\title{
Casuarina species in Algeria: Reviewing identity, distribution and symbiotic status
}

\section{Kathia BELAID ${ }^{1}$ \\ LUke J. POTGIETER ${ }^{2}$ \\ Said AMRANI ${ }^{1}$ \\ Madjid Zızı' ${ }^{1}$ \\ Hassen GHERB $\left.\right|^{3}$}

${ }^{1}$ Université des Sciences

et de la Technologie Houari Boumediène (USTHB)

Faculté des Sciences Biologiques Laboratoire de Biologie

et de Physiologie des Organismes BP 32 El Alia, Alger

Algérie

${ }^{2}$ University of Toronto-Scarborough Department of Biological Sciences 1265 Military Trail

Toronto, ON, M1C 1A4

Canada

${ }^{3}$ Institut de Recherche pour le Développement (IRD) UMR PHIM (IRD/CIRAD/INRAe/

Université de Montpellier/

L'Institut Agro Montpellier)

Campus International de Baillarguet TA A-82/J, 34398 Montpellier Cedex 5 France

Auteur correspondant / Corresponding author:

Hassen GHERBI - hassen.gherbi@ird.fr

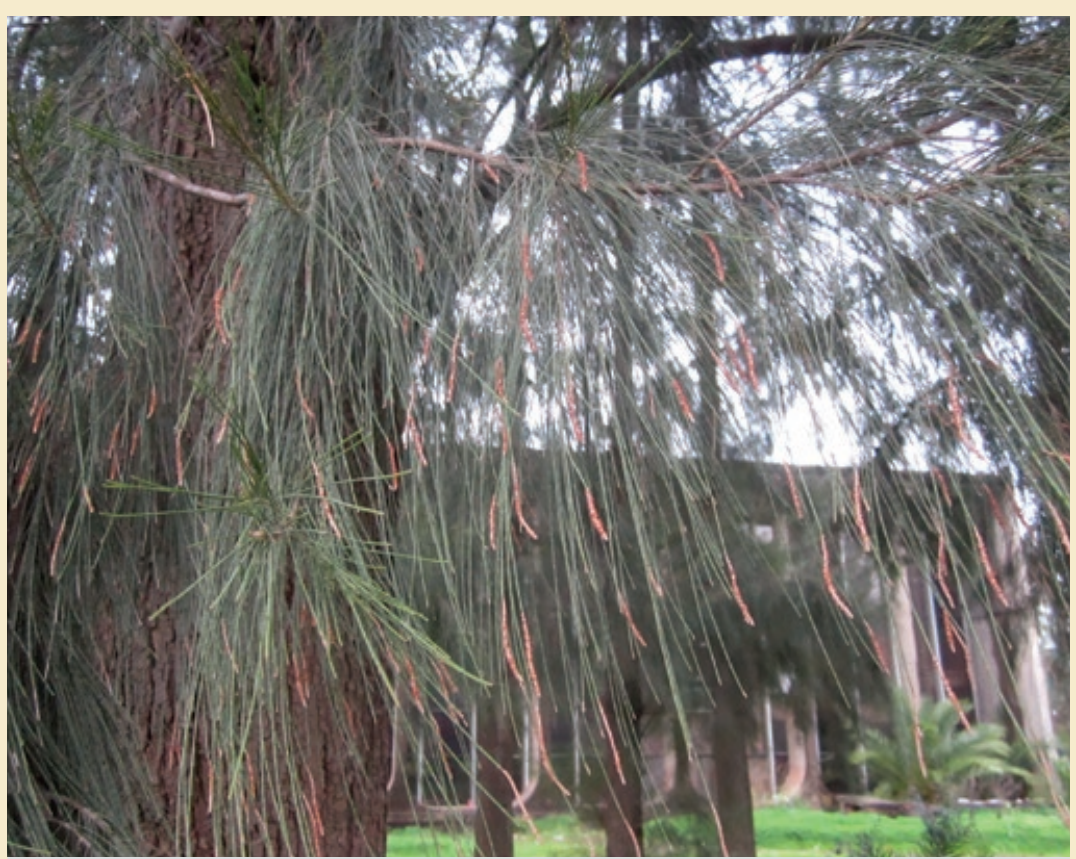

Photos 1.

Utilization of Casuarina trees in Algeria, C. cunninghamiana tree at the North region of Algeria (campus of Houari Boumedienne university, USTHB, district of Algiers). Photo K. Belaid.

Doi : 10.19182/bft2022.351.a36386 - Droit d'auteur ๔ 2022, Bois et Forêts des Tropiques - ๔ Cirad - Date de soumission : 19 mai 2021 ; date d'acceptation : 15 octobre 2021 ; date de publication : $1^{\text {er février } 2022 .}$
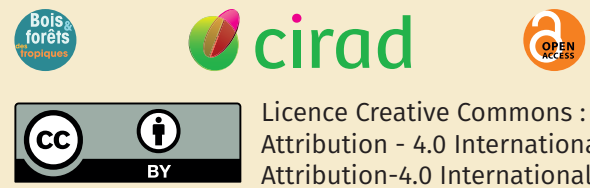

Citer l'article / To cite the article

Belaid K., Potgieter L. J., Amrani S., Zizi M., Gherbi H., 2022. Casuarina species in Algeria: Reviewing identity, distribution and symbiotic status. Bois et Forêts des Tropiques, 351 : 15-28. Doi : https://doi.org/10.19182/bft2022.351. $\underline{\mathrm{a} 36386}$ 


\section{RÉSUMÉ}

\section{Espèces de Casuarina en Algérie : revisiter l'identité, la distribution et le statut symbiotique}

Malgré l'importance prise par les Casuarina en Algérie depuis leur introduction au début du $19^{\mathrm{e}}$ siècle, le nombre d'espèces aujourd'hui présentes dans le pays, leur identité, leur distribution ainsi que leur statut symbiotique sont encore largement méconnus. Une étude menée sur 2304 arbres dans 96 peuplements répartis dans toute l'Algérie indique qu'ils sont représentés exclusivement par cinq espèces du genre Casuarina : Casuarina cristata Miq., C. cunninghamiana Miq., C. equisetifolia L., C. glauca Sieber ex Spreng. et $C$. junghuhniana Miq. Deux espèces, $C$. cunninghamiana et C. glauca, sont largement prédominantes et différemment réparties, la première étant plus fréquente dans les zones relativement humides et tempérées du pays tandis que la seconde est présente dans les régions les plus arides et les plus chaudes. La production de plants de $C$. cunninghamiana et $C$. glauca dans 12 pépinières situées dans le nord et le sud du pays indique qu'ils présentent de faibles taux de mycorhization et de nodulation spontanée. Cela suggère qu'il est nécessaire d'inoculer les jeunes plants avec des souches sélectionnées de champignons mycorhiziens et/ou de Frankia au sein des pépinières afin d'améliorer leur installation durable sur le terrain. Cette étude constitue le premier inventaire de la répartition géographique des Casuarina en Algérie et peut conduire à une meilleure exploitation des arbres de ce genre largement utilisé en Algérie.

Mots-clés : Casuarina, diversité, distribution, mycorhizes, Frankia, nodulation, Algérie.

\section{ABSTRACT}

\section{Casuarina species in Algeria: \\ Reviewing their identity, distribution and symbiotic status}

Since their introduction at the beginning of the $19^{\text {th }}$ century, Casuarina species have become important components of the Algerian landscape, yet the numbers of species present in Algeria and their identity, distribution, and symbiotic status are still not well understood. A survey conducted on 2,304 trees in 96 Casuarina stands across Algeria indicates that they are represented exclusively by five species: Casuarina cristata Miq., C. cunninghamiana Miq., C. equisetifolia L., C. glauca Sieber ex Spreng. and C. junghuhniana Miq. Two species, C. cunninghamiana and C. glauca, were the most common - the former occurring more often in the relatively humid and temperate areas of the country while the latter is most frequent in the drier and warmer areas. Production of seedlings of C. cunninghamiana and C. glauca in 12 nurseries resulted in low rates of spontaneous mycorrhizal infection frequency and nodulation, suggesting that inoculation of seedlings with selected mycorrhizal fungi and/or Frankia strains in the nursery would be necessary to improve their establishment in the field. This study is the first survey of the geographical distribution of Casuarina species in Algeria and should lead to more efficient utilization of this widely used tree genus in Algeria.

Keywords: Casuarina trees, diversity, distribution, mycorrhizae, Frankia, nodulation, Algeria.

\section{RESUMEN}

\author{
Especies de Casuarina en Argelia: \\ estudio de su identidad, distribución \\ y estatus simbiótico
}

A pesar de la importancia de la Casuarina en Argelia desde su introducción a principios del siglo XIX, el número de especies actualmente presentes en el país, su identidad, su distribución, así como su estatus simbiótico son todavía muy desconocidos. Un estudio realizado a 2304 árboles en 96 rodales de toda Argelia indica que están representados exclusivamente por 5 especies del género Casuarina: Casuarina cristata Miq, C. cunninghamiana Miq, C. equisetifolia L., C. glauca Sieber ex Spreng. y C. junghuhniana Miq. Dos especies, C. cunninghamiana y C. glauca, predominan ampliamente y se distribuyen de forma diferente, siendo la primera más común en las zonas relativamente húmedas y templadas del país, mientras que la segunda está presente en las regiones más áridas y cálidas. La producción de plantas de C. cunninghamiana y C. glauca en 12 viveros del norte y el sur del país indica que tienen bajas tasas de micorrización y nodulación espontánea. Esto sugiere que es necesario inocular las plántulas con cepas seleccionadas de hongos micorrícicos y/o de Frankia en el vivero para mejorar su establecimiento duradero en el terreno. Este estudio constituye el primer inventario de la distribución geográfica de la Casuarina en Argelia y puede conducir a una mejor explotación de los árboles de este género, ampliamente utilizados en Argelia.

Palabras clave: Casuarina, diversidad, distribución, micorrizas, Frankia, nodulación, Argelia. 


\section{Introduction}

Casuarina trees are native to Australia, Southeast Asia, and the Pacific Islands (Wheeler et al., 2011). Due to their economic and ecological importance, they have been introduced to over 100 countries around the world with three species (C. cunninghamiana, C. equisetifolia and C. glauca) becoming invasive in many parts of their introduced range (Potgieter et al., 2014).

The Casuarinaceae family consists of approximately 90 species (though this number is still widely debated in the literature) across four genera: Allocasuarina, Casuarina, Ceuthostoma and Gymnostoma (Johnson and Wilson, 1993; Christenhusz and Byng, 2016). The Casuarina genus comprises around 14 species (19 taxa) (Potgieter et al., 2014). They are evergreen, monoecious, or dioecious shrubs or tall trees that resemble conifers by their growth habit and shape, needle-like leaves, and cone-shaped woody fruits. Despite this resemblance which made them considered in the past as gymnosperms, they are a group of dicotyledonous angiosperms (Eudicots) classed in the order Fagales and the Rosids group as defined by APG IV (APG, 2016).

Casuarinas are fast-growing, resilient trees that tolerate harsh habitats and many soil types including some of the most inhospitable (NRC, 1984). They have a fair resistance to heat, drought, salinity, wind, and pests and produce good quality timber of high energy value (Benge, 1982; Dawson, 2008). These adaptations are enhanced by their ability to form multi-beneficial mutualistic symbiosis with mycorrhizal fungi and the actinobacteria Frankia leading to the formation of nitrogen-fixing root nodules. Thus, these remarkable properties make them pioneer species that are capable of colonizing and enriching poor soils and barren sites, thereby playing a particularly important role in ecological succession (Wall, 2000; Duponnois et al., 2003). Commercially important in reforestation, carbon sequestration, agroforestry, and land rehabilitation programs, casuarinas are now common features in many landscapes around the world (Zhong et al., 2010; Potgieter et al., 2014).

Casuarinas were introduced to Algeria in the first half of the $19^{\text {th }}$ century (around 1835). Four species of Casuarina (C. cunninghamiana, C. equisetifolia, C. glauca and C. junghuhniana) and four species of Allocasuarina (A. littoralis, A. paludosa, A. torulosa and $A$. verticillata) were introduced to Algeria during the French colonization (GGA, 1850, 1865; Trottier, 1872). Since then, they have been continuously propagated at a large scale (Toth, 1965; Houmani, 1997) and have today become a fundamental part of the woody flora in all bioclimatic zones of Algeria (Toth, 1965). They are extensively planted for ornamental purposes along roadsides and as windbreaks, fences or shelterbelts protecting against wind, erosion, sand shifting and rapid drying of irrigated fields, particularly in the southern part of the country (Toth, 1965; Houmani, 1997; Gherbi et al., 2011; Makhlouf et al., 2012). They are also used for reforestation, land reclamation of barren or polluted areas and to fight against desertification (Gherbi et al., 2011; Maity and Pawlowski, 2021). Their branchlets with teeth-like leaves are used as fodder and organic matter for soil improvement, and their wood is used for firewood, charcoal production or timber (Bensaid, 1995). In addition, they are a source of food, habitat, refuges, and nest material for many species of insects, birds, and small mammals and thus support and even increase the biodiversity in the areas where they are established (Tarai and Doumandji, 2009).

Although Casuarina trees are widespread over the country, they rarely disperse naturally and are propagated almost exclusively by the transplantation of individuals produced in nurseries from seed germination or to a lesser extent by cuttings (Houmani, 1997). As a result, few naturalized Casuarina populations occur in the country, which might be explained by the more adverse climatic conditions in Algeria compared to their native range (Potgieter et al., 2014).

Despite their considerable importance and increasing application in extensive rehabilitation programs of Saharan lands, the number, identity, importance, and distribution of Casuarina species present in Algeria remain largely unexplored.

In this work, we established a comprehensive inventory and distribution of the members of Casuarina trees throughout Algerian rangelands. We also assessed the occurrence and frequency of the mycorrhization, and the nodulation of Casuarina species cultivated in nurseries across the country. Indeed, since propagation of these trees is done exclusively from nursery stock, the mycorrhization and nodulation of seedlings at the nursery level are crucial both for the successful establishment of the species after transfer into plantation areas and for taking full advantage of their ability to fix nitrogen and to improve soil fertility. This work constitutes the first survey on this family of plants in Algeria and provides valuable data for their utilization in the future.

\section{Materials and Methods}

\section{Survey of Casuarina trees in Algerian rangelands}

To identify the Casuarina species present in Algeria, we analyzed plant material collected during the fall of 2014 and 2015 between September and November from 2,304 trees growing in 96 stands of more than 100 individuals of similar size and age (approximately 10-15 meters and 10-30 years), distributed among all the 48 administrative districts of Algeria (figure 1 and table I for GPS coordinates). From each stand, we sampled three branches bearing cones filled with seeds from 24 randomly selected trees. The Random Number Generator v.1.4 software (2XDSOFT) was used to randomly select individuals in each stand. The collected materials were put in large paper envelopes and taken to the laboratory for examination. For each sampled branch, seven measurements were performed on the selected characters (branch length, article length, teeth number and length, stalk length, cone length and diameter). During our prospection, we did not observe Casuarina trees that were monoecious with male and female flowers borne on the same individual. All the examined trees were dioecious with male and female flowers on separated individuals. 


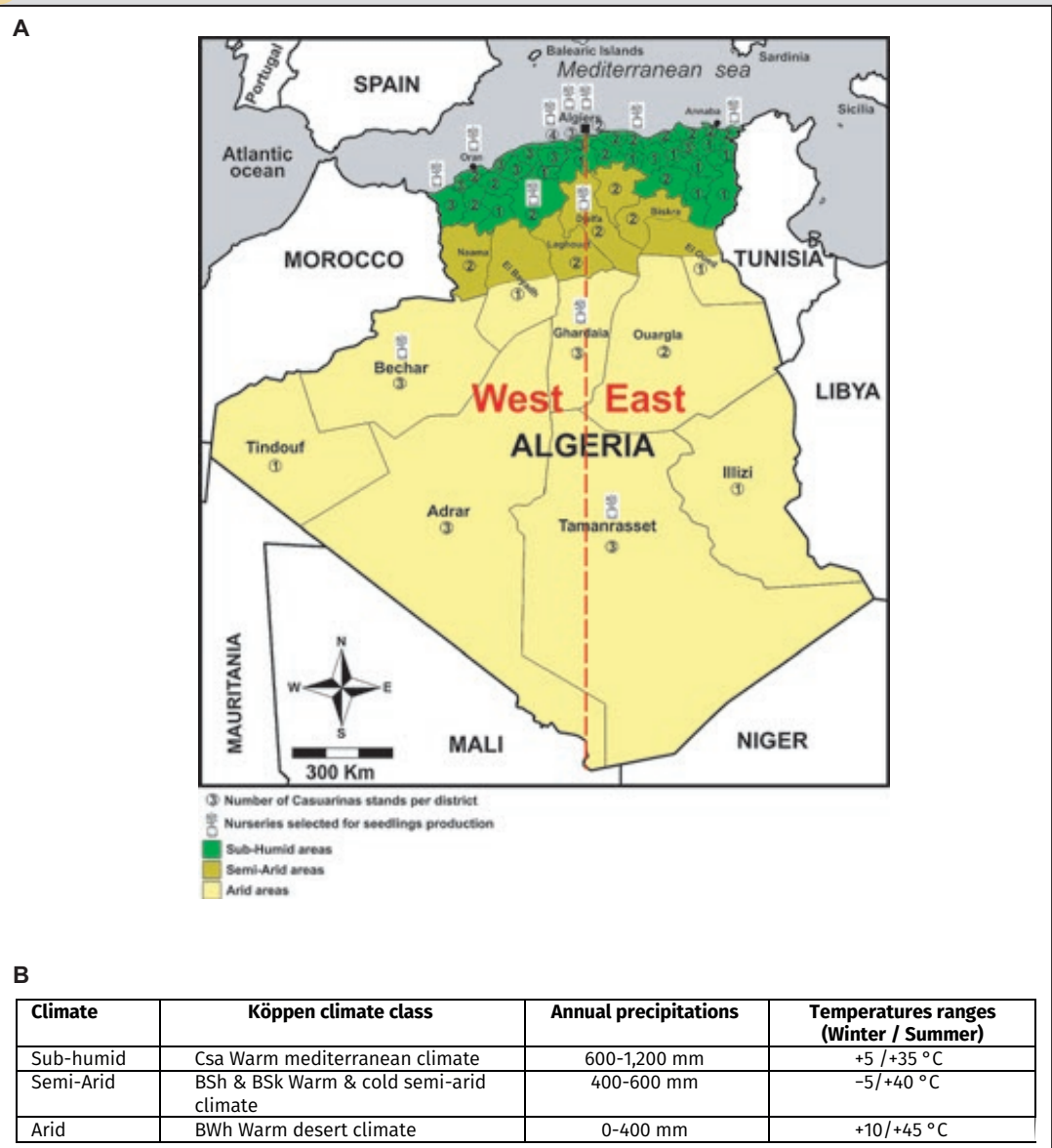

Figure 1.

A. Geographical situation of the Casuarina stands and nurseries selected to perform the study. Numbers surrounded by circles represent the number of stands examined for each of the 48 administrative districts of the country. B. Main characteristics of the 3 major climatic zones of Algeria (source: https://fr.climate-data.org/afrique/ algerie-164/).

\section{Symbiotic status of Casuarina grown in nurseries}

The occurrence of mycorrhizal and actinorhizal symbioses amongst $C$. cunninghamiana and $C$. glauca, which were found to be the most abundant species in Algeria (see Results), was studied. For this purpose, 12-month-old seedlings of each studied species were produced in 12 selected nurseries from the North and the South of Algeria which regularly produce Casuarina seedlings for the local market (figure 1). The seedlings were produced from seeds collected on accurately identified trees growing either in Baïnem arboretum (district of Algiers) for C. cunninghamiana or in the district of Tamanrasset for C. glauca. For each nursery, plants were analyzed for both mycorrhization and nodulation (24 individuals/species). Germination for young trees production was carried out in March 2015 and symbiotic status were examined in March 2016. The seeds were surface-sterilized by immersion in $5 \%$ calcium hypochlorite solution (bleach) for $10 \mathrm{~min}$, rinsed with sterile distilled water and sown on a mixture of nursery soil and thoroughly washed river black sand (2/1: v/v) in perforated polyethylene bags $(15 \times 25 \mathrm{~cm})$ until they reached 12-month-old.

\section{Mycorrhizal symbiosis}

Root systems of seedlings were examined with the naked eye and under a binocular magnifier for the presence of a Hartig net or a hyphal mantle indicating ectomycorrhizal infection (Duponnois et al., 2003). After removing the nodules, the roots of each seedling were excised and stored in ethanol $70^{\circ}$ until use. Afterwards, the presence of endomycorrhizal infections was checked and assessed using the method of Phil-

\section{Casuarina identification}

Casuarina species were identified by their morphometric traits according to the determination key of Wilson and Johnson (1989). Thereby, different traits were considered: the number of sheath teeth (true leaves), the length of branchlets and internodes for the vegetative part, the sizes of inflorescences, cones, and seeds for the floral organs. Macroscopic measurements (length of branchlets, internodes, peduncles, and cones as well as the diameter of cones) were made by using a precision ruler. Microscopic observations and measurements (number and length of teeth by whorls) were carried out under a binocular magnifier (Leica EZ4HD) or a microscope (Premiere MIS-6000T) using (when necessary) a microscope micrometer slide (OPTIKA M-005). However, it should be noted that $0.7 \%$ of the individuals (17 of the 2,304 individuals) collected could not be clearly identified on the basis of these morphometric traits, so that they were excluded from the analysis. lips and Hayman (1970). For each seedling, five thin roots were randomly chosen, cut into $1 \mathrm{~cm}$ segments, and then cleared by soaking in $\mathrm{KOH}(10 \%)$ at $90{ }^{\circ} \mathrm{C}$ for one hour followed by soaking in $\mathrm{H}_{2} \mathrm{O}_{2}$ (9\%) for $30 \mathrm{~min}$. Root segments were thoroughly rinsed with distilled water, soaked in $\mathrm{HCl}$ (1\%) for five minutes and stained with Trypan blue $(0.05 \%$ Trypan blue in lactophenol (phenol/lactic acid v/v) for $20 \mathrm{~min}$ at $90^{\circ} \mathrm{C}$. After removing dye excess by distilled water, segments were incubated in lactophenol for $24 \mathrm{~h}$ to enhance their discoloration. To check for the presence/occurrence of dark blue-coloured fungal structures (hyphae, arbuscules, vesicles and spores), 10 stained segments per root (50 root fragments/seedling) were mounted between a microscopic slide and coverslip in a drop of a glycerol solution (20\%) and examined under an optical microscope. Endomycorrhizal colonization was scored when fungal structures were found in at least one root segment. The intensity of the mycorrhization (I\%) was assessed by counting the number of colonized fragments on the total of roots examined following the 
Table I.

GPS coordinates of the 96 stands from which samples were collected.

\begin{tabular}{|c|c|c|c|}
\hline Locality & Latitude & Longitude & Elevation \\
\hline Adrar - Sali & $26^{\circ} 57^{\prime} 46,44^{\prime \prime} \mathrm{N}$ & $0^{\circ} 01^{\prime} 45,14^{\prime \prime} 0$ & 217 \\
\hline Adrar - Sbaa & $28^{\circ} 13^{\prime} 25,09^{\prime \prime} \mathrm{N}$ & $0^{\circ} 10^{\prime} 17,27 " 0$ & 263 \\
\hline Adrar - Tamantit & $27^{\circ} 44^{\prime} 39,84^{\prime \prime} \mathrm{N}$ & $0^{\circ} 15^{\prime} 45,45^{\prime \prime} 0$ & 244 \\
\hline Ain Defla - Ain Defla & $36^{\circ} 15^{\prime} 11,73^{\prime \prime} \mathrm{N}$ & $2^{\circ} 25^{\prime} 39,01^{\prime \prime} \mathrm{E}$ & 248 \\
\hline Ain Defla - El Attaf & $36^{\circ} 13^{\prime} 30,07^{\prime \prime} \mathrm{N}$ & $1^{\circ} 41^{\prime} 12,39^{\prime \prime} \mathrm{E}$ & 165 \\
\hline Ain Defla - Khemis Miliana & $36^{\circ} 15^{\prime} 00,81^{\prime \prime} \mathrm{N}$ & $2^{\circ} 13^{\prime} 42,08^{\prime \prime} \mathrm{E}$ & 282 \\
\hline Ain Temouchent - Ain Tolba & $35^{\circ} 15^{\prime} 51,73^{\prime \prime} \mathrm{N}$ & $1^{\circ} 16^{\prime} 00,02^{\prime \prime} 0$ & 205 \\
\hline Ain Temouchent - El Malah & $35^{\circ} 23^{\prime} 47,47^{\prime \prime} \mathrm{N}$ & $1^{\circ} 04^{\prime} 15,51^{\prime \prime} 0$ & 65 \\
\hline Ain Temouchent - Terga & $35^{\circ} 25^{\prime} 23,72^{\prime \prime} \mathrm{N}$ & $1^{\circ} 09^{\prime} 38,53^{\prime \prime} 0$ & 51 \\
\hline Algiers - Bainem (INRF) & $36^{\circ} 48^{\prime} 14,30^{\prime \prime} \mathrm{N}$ & $2^{\circ} 57^{\prime} 28,03^{\prime \prime} \mathrm{E}$ & 177 \\
\hline Algiers - Ben Aknoun (Zoo parc) & $36^{\circ} 45^{\prime} 04,36^{\prime \prime} \mathrm{N}$ & $3^{\circ} 00^{\prime} 56,94^{\prime \prime} \mathrm{E}$ & 253 \\
\hline Algiers - USTHB & $36^{\circ} 42^{\prime} 40,65^{\prime \prime} \mathrm{N}$ & $3^{\circ} 42^{\prime} 04,47^{\prime \prime} \mathrm{E}$ & 14 \\
\hline Algiers - Zeralda (F. des planteurs) & $36^{\circ} 42^{\prime} 40,24^{\prime \prime} \mathrm{N}$ & $2^{\circ} 51^{\prime} 30,20^{\prime \prime} \mathrm{E}$ & 63 \\
\hline Annaba - Ben Mhidi & $36^{\circ} 45^{\prime} 14,57^{\prime \prime} \mathrm{N}$ & $7^{\circ} 55^{\prime} 17,67^{\prime \prime} \mathrm{E}$ & 7 \\
\hline Annaba - Chabbia & $36^{\circ} 51^{\prime} 04,52^{\prime \prime} \mathrm{N}$ & $7^{\circ} 42^{\prime} 49,90^{\prime \prime} \mathrm{E}$ & 6 \\
\hline Batna - Bousalh & $35^{\circ} 12^{\prime} 31,73^{\prime \prime} \mathrm{N}$ & $6^{\circ} 17^{\prime} 47,86^{\prime \prime} \mathrm{E}$ & 1064 \\
\hline Batna - Tidjdad & $35^{\circ} 15^{\prime} 52,15^{\prime \prime} \mathrm{N}$ & $6^{\circ} 05^{\prime} 29,13^{\prime \prime} \mathrm{E}$ & 1225 \\
\hline Bechar - Abadla & $31^{\circ} 00^{\prime} 34,75^{\prime \prime} \mathrm{N}$ & $2^{\circ} 44^{\prime} 27,09^{\prime \prime} 0$ & 584 \\
\hline Bechar - Kenadsa & $31^{\circ} 33^{\prime} 54,47^{\prime \prime} \mathrm{N}$ & $2^{\circ} 22^{\prime} 25,29^{\prime \prime} 0$ & 763 \\
\hline Bechar - Ouakda & $31^{\circ} 39^{\prime} 31,74^{\prime \prime} \mathrm{N}$ & $2^{\circ} 10^{\prime} 29,98^{\prime \prime} 0$ & 808 \\
\hline Bejaia - Djebira & $36^{\circ} 42^{\prime} 05,72^{\prime \prime} \mathrm{N}$ & $5^{\circ} 04^{\prime} 14,44^{\prime \prime} \mathrm{E}$ & 36 \\
\hline Bejaia - Oued Ghir & $36^{\circ} 42^{\prime} 15,22^{\prime \prime} \mathrm{N}$ & $4^{\circ} 59^{\prime} 01,21^{\prime \prime} \mathrm{E}$ & 15 \\
\hline Biskra - Doucen & $34^{\circ} 37^{\prime} 48,01^{\prime \prime} \mathrm{N}$ & $4^{\circ} 58^{\prime} 47,53^{\prime \prime} \mathrm{E}$ & 223 \\
\hline Biskra - Ouled Djellal & $34^{\circ} 24^{\prime} 37,07^{\prime \prime} \mathrm{N}$ & $5^{\circ} 04^{\prime} 53,49^{\prime \prime} \mathrm{E}$ & 183 \\
\hline Blida - Boufarik & $36^{\circ} 34^{\prime} 31,15^{\prime \prime} \mathrm{N}$ & $2^{\circ} 57^{\prime} 10,57^{\prime \prime} \mathrm{E}$ & 48 \\
\hline Blida - Meftah (Zayane) & $36^{\circ} 38^{\prime} 03,74^{\prime \prime} \mathrm{N}$ & $3^{\circ} 16^{\prime} 22,69^{\prime \prime} \mathrm{E}$ & 82 \\
\hline Blida - Sidi Moussa & $36^{\circ} 36^{\prime} 09,85^{\prime \prime} \mathrm{N}$ & $3^{\circ} 06^{\prime} 51,13^{\prime \prime} \mathrm{E}$ & 54 \\
\hline Bordj Bou Arreridj - Medjana & $36^{\circ} 08^{\prime} 12,67^{\prime \prime} \mathrm{N}$ & $4^{\circ} 41^{\prime} 10,20^{\prime \prime} \mathrm{E}$ & 1061 \\
\hline Bordj Mokhtar city & $21^{\circ} 19^{\prime} 41,44^{\prime \prime} \mathrm{N}$ & $0^{\circ} 58^{\prime} 19,64^{\prime \prime} \mathrm{E}$ & 400 \\
\hline Bouira - Ain Bessem & $36^{\circ} 20^{\prime} 49,95^{\prime \prime} \mathrm{N}$ & $3^{\circ} 42^{\prime} 22,99^{\prime \prime} \mathrm{E}$ & 738 \\
\hline Bouira - Said Abid & $36^{\circ} 21^{\prime} 08,02^{\prime \prime} \mathrm{N}$ & $3^{\circ} 50^{\prime} 36,97^{\prime \prime} \mathrm{E}$ & 525 \\
\hline Boumerdès - Dellys & $36^{\circ} 54^{\prime} 23,65^{\prime \prime} \mathrm{N}$ & $3^{\circ} 53^{\prime} 36,67^{\prime \prime} \mathrm{E}$ & 335 \\
\hline Boumerdès - Tadkempt & $36^{\circ} 54^{\prime} 07,56^{\prime \prime} \mathrm{N}$ & $3^{\circ} 51^{\prime} 43,34^{\prime \prime} \mathrm{E}$ & 32 \\
\hline Boumerdès - Zaouia & $36^{\circ} 53^{\prime} 00,49^{\prime \prime} \mathrm{N}$ & $3^{\circ} 58^{\prime} 42,94^{\prime \prime} \mathrm{E}$ & 193 \\
\hline Chlef - Boukadir & $36^{\circ} 04^{\prime} 02,80^{\prime \prime} \mathrm{N}$ & $1^{\circ} 08^{\prime} 17,31^{\prime \prime} \mathrm{E}$ & 83 \\
\hline Chlef - Oued Fodda & $36^{\circ} 11^{\prime} 22,87^{\prime \prime} \mathrm{N}$ & $1^{\circ} 31^{\prime} 27,35^{\prime \prime} \mathrm{E}$ & 162 \\
\hline Djelfa - Ain Oussara & $35^{\circ} 28^{\prime} 42,35^{\prime \prime} \mathrm{N}$ & $2^{\circ} 53^{\prime} 48,23^{\prime \prime} \mathrm{E}$ & 663 \\
\hline Djelfa - Hassi Bahbah & $35^{\circ} 05^{\prime} 08,49^{\prime \prime} \mathrm{N}$ & $2^{\circ} 59^{\prime} 36,33^{\prime \prime} \mathrm{E}$ & 905 \\
\hline El Bayadh - El Abiodh Sidi Cheikh & $32^{\circ} 51^{\prime} 01,71^{\prime \prime} \mathrm{N}$ & $0^{\circ} 29^{\prime} 50,01^{\prime \prime} \mathrm{E}$ & 889 \\
\hline El Oued - Hazoua & $33^{\circ} 45^{\prime} 20,47^{\prime \prime} \mathrm{N}$ & $7^{\circ} 38^{\prime} 45,19^{\prime \prime} \mathrm{E}$ & 35 \\
\hline El Tarf - Ain Al Assel & $36^{\circ} 46^{\prime} 15,07^{\prime \prime} \mathrm{N}$ & $8^{\circ} 22^{\prime} 05,53^{\prime \prime} \mathrm{E}$ & 30 \\
\hline El Tarf - Boutaldja & $36^{\circ} 48^{\prime} 00,58^{\prime \prime} \mathrm{N}$ & $8^{\circ} 12^{\prime} 06,75^{\prime \prime} \mathrm{E}$ & 12 \\
\hline Ghardaia - El Meniaa & $30^{\circ} 38^{\prime} 49,33^{\prime \prime} \mathrm{N}$ & $2^{\circ} 59^{\prime} 18,83^{\prime \prime} \mathrm{E}$ & 421 \\
\hline Ghardaia - Hassi El Fahl & $31^{\circ} 36^{\prime} 49,52^{\prime \prime} \mathrm{N}$ & $3^{\circ} 40^{\prime} 58,91 " \mathrm{E}$ & 375 \\
\hline Ghardaia - Zelfana & $32^{\circ} 25^{\prime} 48,11^{\prime \prime} \mathrm{N}$ & $4^{\circ} 13^{\prime} 40,17^{\prime \prime} \mathrm{E}$ & 364 \\
\hline Illizi City & $26^{\circ} 28^{\prime} 53,06^{\prime \prime} \mathrm{N}$ & $8^{\circ} 27^{\prime} 34,00^{\prime \prime} \mathrm{E}$ & 556 \\
\hline Jijel - Airport & $36^{\circ} 48^{\prime} 00,43^{\prime \prime} \mathrm{N}$ & $5^{\circ} 52^{\prime} 38,64^{\prime \prime} \mathrm{E}$ & 5 \\
\hline Jijel - Tamila & $36^{\circ} 47^{\prime} 40,74^{\prime \prime} \mathrm{N}$ & $5^{\circ} 51^{\prime} 49,66^{\prime \prime} \mathrm{E}$ & 8 \\
\hline
\end{tabular}


Table I (following).

\begin{tabular}{|c|c|c|c|}
\hline Locality & Latitude & Longitude & Elevation \\
\hline Laghouat - Al Assafia & $33^{\circ} 49^{\prime} 53,98^{\prime \prime} \mathrm{N}$ & $2^{\circ} 58^{\prime} 52,08^{\prime \prime} \mathrm{E}$ & 750 \\
\hline Laghouat - El Hadjeb Tadjmout & $33^{\circ} 56^{\prime} 50,75^{\prime \prime} \mathrm{N}$ & $2^{\circ} 38^{\prime} 09,92^{\prime \prime} \mathrm{E}$ & 972 \\
\hline Mascara - Froha & $35^{\circ} 17^{\prime} 47,72^{\prime \prime} \mathrm{N}$ & $0^{\circ} 07^{\prime} 46,36^{\prime \prime} \mathrm{E}$ & 469 \\
\hline Mascara - Ghriss & $35^{\circ} 15^{\prime} 11,04^{\prime \prime} \mathrm{N}$ & $0^{\circ} 10^{\prime} 02,47^{\prime \prime} \mathrm{E}$ & 495 \\
\hline Mascara - Tizi & $35^{\circ} 19^{\prime} 11,04^{\prime \prime} \mathrm{N}$ & $0^{\circ} 04^{\prime} 38,94^{\prime \prime} \mathrm{E}$ & 455 \\
\hline Mila - Sidi Mérouane & $36^{\circ} 30^{\prime} 51,94^{\prime \prime} \mathrm{N}$ & $6^{\circ} 15^{\prime} 45,62^{\prime \prime} \mathrm{E}$ & 342 \\
\hline Mila City & $36^{\circ} 27^{\prime} 17,57^{\prime \prime} \mathrm{N}$ & $6^{\circ} 16^{\prime} 32,43^{\prime \prime} \mathrm{E}$ & 431 \\
\hline Mostaganem - Hassi Mameche & $35^{\circ} 51^{\prime} 42,91^{\prime \prime} \mathrm{N}$ & $0^{\circ} 04^{\prime} 46,06 \mathrm{E}$ & 137 \\
\hline Mostaganem - Mazagran & $35^{\circ} 54^{\prime} 07,58^{\prime \prime} \mathrm{N}$ & $0^{\circ} 05^{\prime} 17,64^{\prime \prime} \mathrm{E}$ & 131 \\
\hline Msila - Bou Saada & $35^{\circ} 15^{\prime} 27,76^{\prime \prime} \mathrm{N}$ & $4^{\circ} 13^{\prime} 36,32^{\prime \prime} \mathrm{E}$ & 501 \\
\hline Msila - Ouled Mahdi & $35^{\circ} 34^{\prime} 07,26^{\prime \prime} \mathrm{N}$ & $4^{\circ} 31^{\prime} 28,74^{\prime \prime} \mathrm{E}$ & 407 \\
\hline Naama - Ain Sefra & $32^{\circ} 44^{\prime} 32,15^{\prime \prime} \mathrm{N}$ & $0^{\circ} 35^{\prime} 36,33^{\prime \prime} 0$ & 1081 \\
\hline Naama City & $33^{\circ} 16^{\prime} 13,89^{\prime \prime} \mathrm{N}$ & $0^{\circ} 18^{\prime} 24,61^{\prime \prime} 0$ & 1171 \\
\hline Oran - Es senia & $35^{\circ} 37^{\prime} 21,52^{\prime \prime} \mathrm{N}$ & $0^{\circ} 40^{\prime} 19,460$ & 90 \\
\hline Oran - Messerghine & $35^{\circ} 37^{\prime} 02,82^{\prime \prime} \mathrm{N}$ & $0^{\circ} 44^{\prime} 31,56^{\prime \prime} 0$ & 125 \\
\hline Ouargla - Haoud El Hamra & $31^{\circ} 53^{\prime} 28,69^{\prime \prime} \mathrm{N}$ & $5^{\circ} 28^{\prime} 24,17 " E$ & 153 \\
\hline Ouargla - Hassi Ben Abdelah & $32^{\circ} 11^{\prime} 55,52^{\prime \prime} \mathrm{N}$ & $5^{\circ} 42^{\prime} 46,37^{\prime \prime} \mathrm{E}$ & 155 \\
\hline Relizane - Belassel Bouzegza & $35^{\circ} 50^{\prime} 21,34^{\prime \prime} \mathrm{N}$ & $0^{\circ} 29^{\prime} 24,11^{\prime \prime} \mathrm{E}$ & 67 \\
\hline Relizane - Chaif El Hassene & $35^{\circ} 40^{\prime} 58,85^{\prime \prime} \mathrm{N}$ & $0^{\circ} 33^{\prime} 50,04^{\prime \prime} \mathrm{E}$ & 90 \\
\hline Relizane - Matmar & $35^{\circ} 43^{\prime} 45,72^{\prime \prime} \mathrm{N}$ & $0^{\circ} 28^{\prime} 29,28^{\prime \prime} \mathrm{E}$ & 69 \\
\hline Saida - Ain El Hadjar & $34^{\circ} 45^{\prime} 13,68^{\prime \prime} \mathrm{N}$ & $0^{\circ} 08^{\prime} 40,22^{\prime \prime} \mathrm{E}$ & 1025 \\
\hline Sidi Bel Abbès - Airport & $35^{\circ} 10^{\prime} 29,28^{\prime \prime} \mathrm{N}$ & $0^{\circ} 35^{\prime} 40,20^{\prime \prime} 0$ & 481 \\
\hline Sidi Bel Abbès - Sidi Brahim & $35^{\circ} 16^{\prime} 01,99^{\prime \prime} \mathrm{N}$ & $0^{\circ} 34^{\prime} 26,26^{\prime \prime} 0$ & 433 \\
\hline Sidi Bel Abbès - Tilmouni & $35^{\circ} 10^{\prime} 36,43^{\prime \prime} \mathrm{N}$ & $0^{\circ} 31^{\prime} 26,75^{\prime \prime} 0$ & 510 \\
\hline Sétif - Fermatou & $36^{\circ} 13^{\prime} 33,67^{\prime \prime} \mathrm{N}$ & $5^{\circ} 23^{\prime} 46,88^{\prime \prime} \mathrm{E}$ & 1048 \\
\hline Skikda - Hamadi Krouma & $36^{\circ} 50^{\prime} 55,24^{\prime \prime} \mathrm{N}$ & $6^{\circ} 54^{\prime} 15,73^{\prime \prime} \mathrm{E}$ & 30 \\
\hline Skikda - Larbi Ben Mhidi & $36^{\circ} 52^{\prime} 45,26^{\prime \prime} \mathrm{N}$ & $7^{\circ} 00^{\prime} 11,20^{\prime \prime} \mathrm{E}$ & 88 \\
\hline Tamanrasset - Djanet & $24^{\circ} 33^{\prime} 29,96^{\prime \prime} \mathrm{N}$ & $9^{\circ} 28^{\prime} 58,16^{\prime \prime} \mathrm{E}$ & 1041 \\
\hline Tamanrasset - Idles & $23^{\circ} 49^{\prime} 38,29^{\prime \prime} \mathrm{N}$ & $5^{\circ} 55^{\prime} 53,70^{\prime \prime} \mathrm{E}$ & 1390 \\
\hline Tamanrasset City & $22^{\circ} 46^{\prime} 56,36^{\prime \prime} \mathrm{N}$ & $5^{\circ} 33^{\prime} 50,64^{\prime \prime} \mathrm{E}$ & 1396 \\
\hline Tebessa - El Ma Labiodh & $35^{\circ} 17^{\prime} 30,68^{\prime \prime} \mathrm{N}$ & $8^{\circ} 11^{\prime} 26,13^{\prime \prime} \mathrm{E}$ & 1103 \\
\hline Tebessa - Oum Ali & $35^{\circ} 00^{\prime} 32,96^{\prime \prime} \mathrm{N}$ & $8^{\circ} 15^{\prime} 15,11 " \mathrm{E}$ & 916 \\
\hline Tiaret - Frenda & $35^{\circ} 03^{\prime} 14,05^{\prime \prime} \mathrm{N}$ & $1^{\circ} 01^{\prime} 26,86^{\prime \prime} \mathrm{E}$ & 837 \\
\hline Tiaret - Sougueur & $35^{\circ} 10^{\prime} 50,02^{\prime \prime} \mathrm{N}$ & $1^{\circ} 28^{\prime} 44,64^{\prime \prime} \mathrm{E}$ & 1150 \\
\hline Tindouf - Aéroport & $27^{\circ} 41^{\prime} 49,43^{\prime \prime} \mathrm{N}$ & $8^{\circ} 09^{\prime} 19,68^{\prime \prime} \mathrm{E}$ & 438 \\
\hline Tipaza Cherchell & $36^{\circ} 36^{\prime} 13,86^{\prime \prime} \mathrm{N}$ & $2^{\circ} 13^{\prime} 01,61^{\prime \prime} \mathrm{E}$ & 148 \\
\hline Tipaza Hadjout & $36^{\circ} 32^{\prime} 09,01^{\prime \prime} \mathrm{N}$ & $2^{\circ} 25^{\prime} 39,01^{\prime \prime} \mathrm{E}$ & 79 \\
\hline Tipaza Sidi Amar & $36^{\circ} 32^{\prime} 52,99^{\prime \prime} \mathrm{N}$ & $2^{\circ} 18^{\prime} 42,34^{\prime \prime} \mathrm{E}$ & 54 \\
\hline Tissemsilt - City & $35^{\circ} 35^{\prime} 18,05^{\prime \prime} \mathrm{N}$ & $1^{\circ} 49^{\prime} 01,65^{\prime \prime} \mathrm{E}$ & 937 \\
\hline Tizi Ouzou - Azzefoun & $36^{\circ} 53^{\prime} 53,90^{\prime \prime} \mathrm{N}$ & $4^{\circ} 25^{\prime} 27,24^{\prime \prime} \mathrm{E}$ & 79 \\
\hline Tizi Ouzou - M’letta & $36^{\circ} 53^{\prime} 22,71^{\prime \prime} \mathrm{N}$ & $4^{\circ} 22^{\prime} 10,47^{\prime \prime} \mathrm{E}$ & 24 \\
\hline Tizi Ouzou - Tigzirt & $36^{\circ} 54^{\prime} 05,29^{\prime \prime} \mathrm{N}$ & $4^{\circ} 09^{\prime} 06,29^{\prime \prime} \mathrm{E}$ & 76 \\
\hline Tlemcen - Hennaya & $34^{\circ} 58^{\prime} 08,84^{\prime \prime} \mathrm{N}$ & $1^{\circ} 22^{\prime} 14,12^{\prime \prime} 0$ & 365 \\
\hline Tlemcen - Maghnia & $35^{\circ} 50^{\prime} 38,57^{\prime \prime} \mathrm{N}$ & $1^{\circ} 42^{\prime} 54,59^{\prime \prime} 0$ & 371 \\
\hline Tlemcen - Taffessera & $35^{\circ} 40^{\prime} 34,89^{\prime \prime} \mathrm{N}$ & $1^{\circ} 26^{\prime} 30,32^{\prime \prime} 0$ & 807 \\
\hline Touggourt - El Arfiane & $33^{\circ} 39^{\prime} 14,96^{\prime \prime} \mathrm{N}$ & $5^{\circ} 58^{\prime} 48,54^{\prime \prime} \mathrm{E}$ & 24 \\
\hline Touggourt - El Mansoura & $33^{\circ} 26^{\prime} 13,07^{\prime \prime} \mathrm{N}$ & $5^{\circ} 58^{\prime} 12,26^{\prime \prime} \mathrm{E}$ & 53 \\
\hline Touggourt - Moggar & $33^{\circ} 13^{\prime} 19,47^{\prime \prime} \mathrm{N}$ & $6^{\circ} 04^{\prime} 56,40^{\prime \prime} \mathrm{E}$ & 61 \\
\hline
\end{tabular}


method described by Trouvelot et al. (1986). This method is based on the range of values from $n 0$ to $n 5$ where $n$ indicates the number of root fragments scored and rating corresponds to: 0 = without colonization of the root segment; 1 = less than $1 \%$ is colonized or colonization trace; 2 = less than $10 \% ; 3=11$ to $50 \% ; 4=51$ to $90 \% ; 5=$ more than $90 \%$. From this, the intensity of mycorrhization is calculated according to the following formula: $1 \%=(95 n 5+70 n 4+30 n 3+5 n 2+n 1) / N$, where $\mathrm{N}$ is the total of observed root fragments.

\section{Actinorhizal symbiosis}

From each of the 12 nurseries investigated, 24 randomly selected plants of C. cunninghamiana or C. glauca were harvested after 12 months of growth and examined in the laboratory. Root systems of the seedlings were carefully removed from the polyethylene bags, washed thoroughly under a stream of tap water to remove soil and then eye examined for the presence of actinorhizal nodules. Nodules of each seedling were excised, and their dry weights were determined after desiccation at $80^{\circ} \mathrm{C}$ until constant weight (1 week).

\section{Statistical analyses}

Canonical Discriminant Analysis (CDA) was performed using the free software Tanagra (Rakotomalala, 2005). CDA is a multivariate statistical method that can highlight differences among groups of individuals (or treatments) and help to better understand of the relationships among the variables measured (branch length, article length, teeth number and length, stalk length, cone length and diameter) within those groups (Cruz-Castillo et al., 1994). We used this method to determine which variables discriminate between two or more naturally occurring groups. Statistical analyses were performed using the free software Past3 (Hammer et al., 2001). Data storage, basic statistics and graphs were performed using Lotus Approach (Lotus Development Corporation, Cambridge, USA).

Data presented in tables II and III were subjected to one-way analyses of variance (ANOVA) and Fisher's Least Significant Difference (LSD) tests to evaluate significant differences for variables at $5 \%$ level while the Pearson's $c^{2}$ test was used to assess the homogeneity of proportions in figures 3 and 4 using R Studio v. 2.13 (R Development Core Team, 2016).

Table II.

Endomycorrhizal symbiosis: Percentage of mycorrhized seedlings and mycorrhizal intensity in root tissues (I\%) among 12-month-old seedlings of Casuarina cunninghamiana and C. glauca produced in 12 nurseries located in the North and the South of Algeria. Data of mycorrhizal intensity (1\%) were subjected to one-way analyses of variance (ANOVA) and Fisher's Least Significant Difference (LSD) tests at $p=0.05$ threshold. Mean values \pm SD $(n=24)$ in each column followed by a different letter are significantly different.

\begin{tabular}{|c|c|c|c|c|}
\hline \multirow[t]{2}{*}{ Nursery localization } & \multicolumn{2}{|c|}{ Casuarina cunninghamiana } & \multicolumn{2}{|c|}{ Casuarina glauca } \\
\hline & $\begin{array}{l}\% \text { of mycorrhized } \\
\text { seedlings }\end{array}$ & $\begin{array}{c}\text { Mycorrhizal } \\
\text { intensity (I \%) }\end{array}$ & $\begin{array}{l}\% \text { of mycorrhized } \\
\text { seedlings }\end{array}$ & $\begin{array}{c}\text { Mycorrhizal } \\
\text { intensity (I \%) }\end{array}$ \\
\hline Algiers - Bainem & 100.0 & $83.1 \pm 2.3^{\mathrm{a}}$ & 100.0 & $83.4 \pm 3.5^{\mathrm{a}}$ \\
\hline Algiers - Ben Aknoun & 100.0 & $79.4 \pm 3.3^{\mathrm{a}}$ & 100.0 & $72.6 \pm 5.4^{\mathrm{a}}$ \\
\hline Béjaïa & 100.0 & $64.8 \pm 4.5^{\mathrm{a}}$ & 83.3 & $60.8 \pm 6.6^{a}$ \\
\hline El Kala & 100.0 & $84.2 \pm 3.5^{\mathrm{a}}$ & 100.0 & $80.8 \pm 5.5^{a}$ \\
\hline Oran & 70.8 & $58.2 \pm 7.1^{a}$ & 100.0 & $58.5 \pm 9.7^{a}$ \\
\hline Tlemcen & 100.0 & $69.8 \pm 1.8^{a}$ & 100.0 & $80.0 \pm 6.5^{b}$ \\
\hline Mean - Northern nurseries & $95.1 \pm 4.9^{a}$ & $73.2 \pm 4.3^{a}$ & $97.2 \pm 2.8^{\mathrm{a}}$ & $72.7 \pm 4.4^{\mathrm{a}}$ \\
\hline Adrar & 79.2 & $33.8 \pm 5.5^{a}$ & 91.7 & $37.0 \pm 5.2^{\mathrm{a}}$ \\
\hline Biskra & 79.2 & $29.4 \pm 6.9^{a}$ & 91.7 & $32.2 \pm 8.8^{\mathrm{a}}$ \\
\hline Djanet & 70.8 & $35.2 \pm 5.0^{\mathrm{a}}$ & 87.5 & $37.5 \pm 7.1^{\mathrm{a}}$ \\
\hline El Golea & 83.3 & $41.2 \pm 9.3^{\mathrm{a}}$ & 100.0 & $44.0 \pm 5.3^{a}$ \\
\hline Tamanrasset & 79.2 & $33.3 \pm 5.2^{\mathrm{a}}$ & 87.5 & $28.7 \pm 7.2^{\mathrm{a}}$ \\
\hline Tindouf & 75.0 & $22.9 \pm 8.3^{a}$ & 83.3 & $24.6 \pm 9.1^{\mathrm{a}}$ \\
\hline Mean - Southern nurseries & $77.8 \pm 1.8^{a}$ & $32.6 \pm 2.5^{a}$ & $90.3 \pm 2.3^{b}$ & $34.0 \pm 2.83^{a}$ \\
\hline Overall mean value & $86.5 \pm 3.6$ & $52.9 \pm 6.5$ & $93.8 \pm 2.0$ & $53.4 \pm 6.3$ \\
\hline
\end{tabular}




\section{Table III.}

Actinorhizal symbiosis: Percentage of nodulated seedlings, mean number of nodules per seedling (nodules/seedling) and nodule dry weight per seedling (NDW mg/seedling) among 12-month-old seedlings of Casuarina cunninghamiana and C. glauca produced in 12 nurseries located in the North and the South of Algeria. Data were subjected to one-way analyses of variance (ANOVA) and Fisher's Least Significant Difference (LSD) tests at $p=0.05$ threshold for all variables. Mean values \pm SD $(n=24)$ in each column followed by a different letter are significantly different.

\begin{tabular}{|c|c|c|c|c|c|c|}
\hline \multirow[t]{2}{*}{ Nursery localization } & \multicolumn{3}{|c|}{ Casuarina cunninghamiana } & \multicolumn{3}{|c|}{ Casuarina glauca } \\
\hline & $\begin{array}{l}\% \text { of nodulated } \\
\text { seedlings }\end{array}$ & $\begin{array}{l}\text { Nodule no./ } \\
\text { seedling }\end{array}$ & $\begin{array}{c}\text { NDW mg/ } \\
\text { seedling }\end{array}$ & $\begin{array}{l}\% \text { of nodulated } \\
\text { seedlings }\end{array}$ & $\begin{array}{c}\text { Nodule no./ } \\
\text { seedling }\end{array}$ & $\begin{array}{l}\text { NDW mg/ } \\
\text { seedling }\end{array}$ \\
\hline Algiers Baïnem & 70.8 & $6.5 \pm 1.0^{\mathrm{a}}$ & $532.7 \pm 81.8^{\mathrm{a}}$ & 54.2 & $4.5 \pm 0.8^{\mathrm{a}}$ & $329.7 \pm 74.5^{b}$ \\
\hline Algiers Ben Aknoun & 37.5 & $1.8 \pm 0.6^{a}$ & $124.4 \pm 35.7^{\mathrm{a}}$ & 41.7 & $3.0 \pm 0.8^{b}$ & $141.7 \pm 38.4^{b}$ \\
\hline Béjaïa & 50.0 & $2.9 \pm 0.6^{a}$ & $243.0 \pm 54.9^{a}$ & 37.5 & $2.6 \pm 0.8^{a}$ & $202.3 \pm 64.3^{a}$ \\
\hline El Kala & 54.2 & $7.0 \pm 1.4^{\mathrm{a}}$ & $278.1 \pm 56.2^{a}$ & 29.2 & $3.0 \pm 1.1^{b}$ & $156.7 \pm 56.7^{a}$ \\
\hline Oran & 33.3 & $2.0 \pm 0.6^{a}$ & $97.2 \pm 30.2^{\mathrm{a}}$ & 45.8 & $2.8 \pm 0.7^{b}$ & $100.8 \pm 26.0^{a}$ \\
\hline Tlemcen & 33.3 & $1.3 \pm 0.5^{a}$ & $33.9 \pm 13.9^{a}$ & 37.5 & $2.0 \pm 0.6^{b}$ & $71.2 \pm 21.5^{b}$ \\
\hline Mean-Northern nurseries & $46.5 \pm 6.0^{\mathrm{a}}$ & $3.6 \pm 1.0^{\mathrm{a}}$ & $218.1 \pm 73.2^{\mathrm{a}}$ & $41.0 \pm 3.5^{\mathrm{a}}$ & $3.0 \pm 0.3^{b}$ & $167.1 \pm 37.4^{b}$ \\
\hline Adrar & 29.2 & $1.1 \pm 0.4^{\mathrm{a}}$ & $16.9 \pm 6.3^{\mathrm{a}}$ & 25.0 & $0.5 \pm 0.2^{b}$ & $12.8 \pm 5.4^{\mathrm{a}}$ \\
\hline Biskra & 20.8 & $1.0 \pm 0.4^{a}$ & $16.2 \pm 7.1^{\mathrm{a}}$ & 25.0 & $0.6 \pm 0.2^{\mathrm{a}}$ & $8.57 \pm 3.4^{b}$ \\
\hline Djanet & 0.0 & $0.0 \pm 0.0^{\mathrm{a}}$ & $0.0 \pm 0.0^{a}$ & 0.0 & $0.0 \pm 0.0^{\mathrm{a}}$ & $0.0 \pm 0.0^{a}$ \\
\hline El Goléa & 0.0 & $0.0 \pm 0.0^{\mathrm{a}}$ & $0.0 \pm 0.0^{a}$ & 0.0 & $0.0 \pm 0.0^{a}$ & $0.0 \pm 0.0^{a}$ \\
\hline Tamanrasset & 0.0 & $0.0 \pm 0.0^{\mathrm{a}}$ & $0.0 \pm 0.0^{\mathrm{a}}$ & 0.0 & $0.0 \pm 0.0^{\mathrm{a}}$ & $0.0 \pm 0.0^{\mathrm{a}}$ \\
\hline Tindouf & 16.7 & $0.5 \pm 0.2^{\mathrm{a}}$ & $6.5 \pm 3.3^{\mathrm{a}}$ & 20.8 & $0.3 \pm 0.2^{\mathrm{a}}$ & $6.9 \pm 3.1^{\mathrm{a}}$ \\
\hline Mean-Southern nurseries & $11.1 \pm 5.2^{\mathrm{a}}$ & $0.4 \pm 0.2^{\mathrm{a}}$ & $6.6 \pm 3.3^{\mathrm{a}}$ & $11.8 \pm 5.3^{\mathrm{a}}$ & $0.2 \pm 0.1^{\mathrm{b}}$ & $4.71 \pm 2.2^{\mathrm{a}}$ \\
\hline Overall mean value & $28.8 \pm 6.5$ & $2.0 \pm 0.7$ & $112.4 \pm 47.3$ & $26.4 \pm 5.3$ & $1.6 \pm 0.4$ & $85.9 \pm 30.3$ \\
\hline
\end{tabular}

\section{Results}

\section{Casuarina species in Algerian rangelands}

Surveys carried out all over Algeria to select sufficiently large and representative tree stands allowed us to observe members of both genera Casuarina and Allocasuarina. Casuarina species are distinguished by their dull and grey to yellow-brown winged seed (samara) body while Allocasuarina species are easily recognizable by their shiny red-brown to black samara body.

In all the prospected locations, the examined stands consisted exclusively of representatives of the genus Casuarina which are widespread across all Algerian rangelands and bioclimatic areas (figure 1, photos 2). In contrast, only one species of Allocasuarina (likely A. verticillata (Lam) L.M.S. Johnson), was recorded in a unique site at the arboretum of Baïnem in Algiers indicating that species of this genus are seemingly rare in Algeria.

\section{Casuarina genus is represented by 5 species in Algeria}

Casuarina plant material was collected from 2,304 randomly selected trees present in 96 stands (24 individuals per stand) located in the 48 administrative districts of the country (figure 1). Analyses based on different morphometric traits of the vegetative parts (sheath teeth, length of branchlets and internodes) and of floral organs (size of cones and seeds) allowed us to identify five species: Casuarina cristata Miq., C. cunninghamiana Miq., C. equisetifolia L., C. glauca Sieber ex Spreng. and C. junghuhniana Miq. (figure 2).

The results of Canonical Discriminant Analysis (CDA) revealed that branch (BR-L) and article (ART-L) lengths were the best discriminating traits since they allow a satisfactory grouping of the individuals from the same species into rather well delineated and cohesive clouds. Nevertheless, except for C. cunninghamiana, all the other groups showed some individuals that were scattered far from the centre of their corresponding cloud (figure 2).

Analysis of the 2,304 plants indicated that the composition of the Casuarina species present in the 96 stands was not homogeneous ( $x^{2}$ test, $p<0.001$ ) since more than half $(55.2 \%)$ included individuals belonging to at least two species (figure 3 ) and nearly $25 \%$ of the stands contained individuals belonging to three species or more. On the other hand, $44.8 \%$ of the stands were monospecific (figure 3 ) and were dominated by either C. cunninghamiana or C. glauca (figure 2, photos 2).

Species of Casuarina that occur in the 96 examined stands are unevenly represented. The proportion 
of each species as well as their distribution in the stands were not homogeneous ( $x^{2}$ test, $p<0.001$ ) (figure 4). Two species, C. cunninghamiana (1,101 individuals) (photos 3 ) and C. glauca (1,070 individuals) (photos 4), were largely predominant accounting together for nearly $95 \%$ of all 2,304 trees examined (figure 4). The two species are also the most widespread since they were found respectively in 68 and 64 of the 96 stands investigated representing $70.8 \%$ and $66.7 \%$ of the cases, respectively (figure 4). The three other Casuarina species, C. cristata (12 individuals), C. equisetifolia (42 individuals) and C. junghuhniana (62 individuals) are much less frequent and represent altogether only $5.0 \%$ of sampled trees (116 out of 2,304). They were found in 4, 16 and 18 stands respectively representing $4.2 \%, 16.7 \%$ and $18.7 \%$ of the 96 stands (figure 4). These species can thus be considered as minor in Algeria. In addition, 17 individuals representing $0.7 \%$ of the total plants could not be identified (figure 4).

Casuarina cunninghamiana is more common in the northern and eastern parts of the country, which are the wettest and most temperate zones, while C. glauca is more abundant in the South and the West, the driest and warmest areas of the country (figure 5).

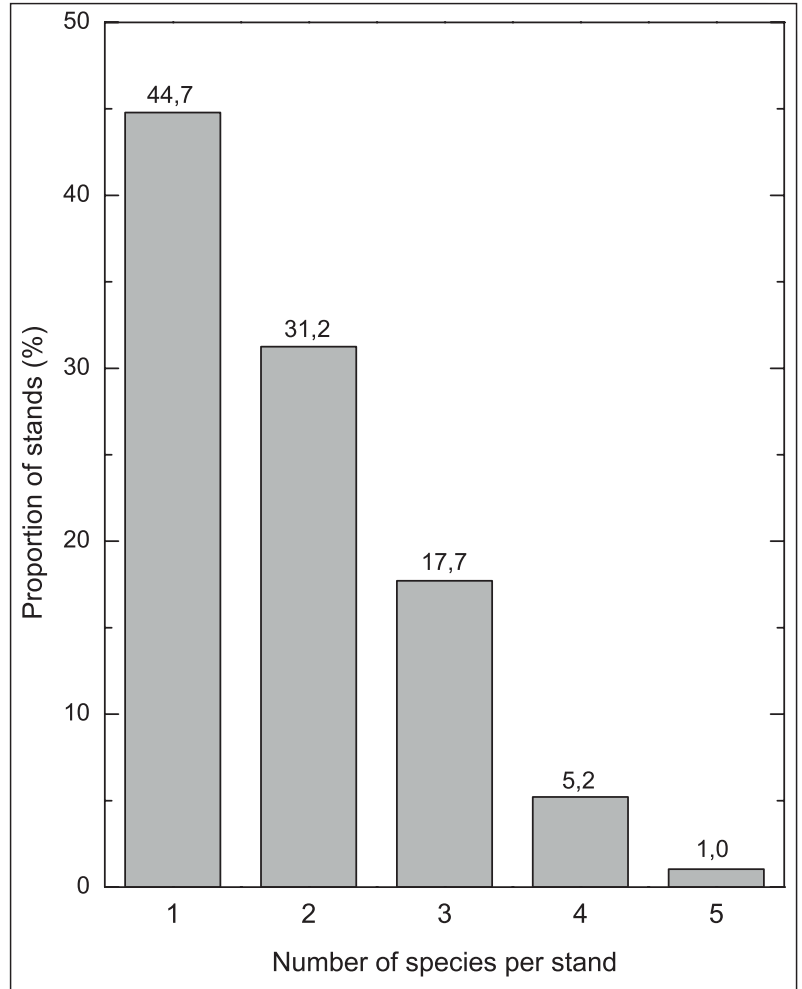

Figure 3.

Distribution of the stands studied according to the number of co-occurring Casuarina species. The composition in Casuarina species present in the 96 stands was not homogeneous $\left(x^{2}\right.$ test: $\left.x_{(4)}^{2}=63.58, p<0.001\right)$.

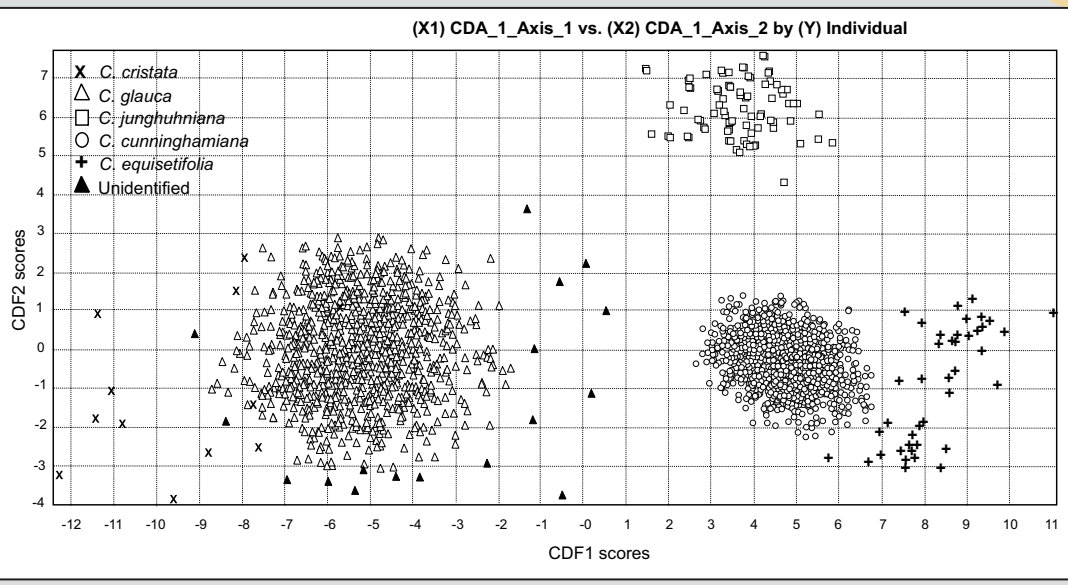

Figure 2.

Canonical scores of the first two canonical discriminant functions (CDFs) of the five species of Casuarina identified on the basis of their morphometric traits. (X) C. cristata,

$(\triangle)$ C. glauca, $(\square)$ C. junghuhniana, (O) C. cunninghamiana, $(+)$ C. equisetifolia.
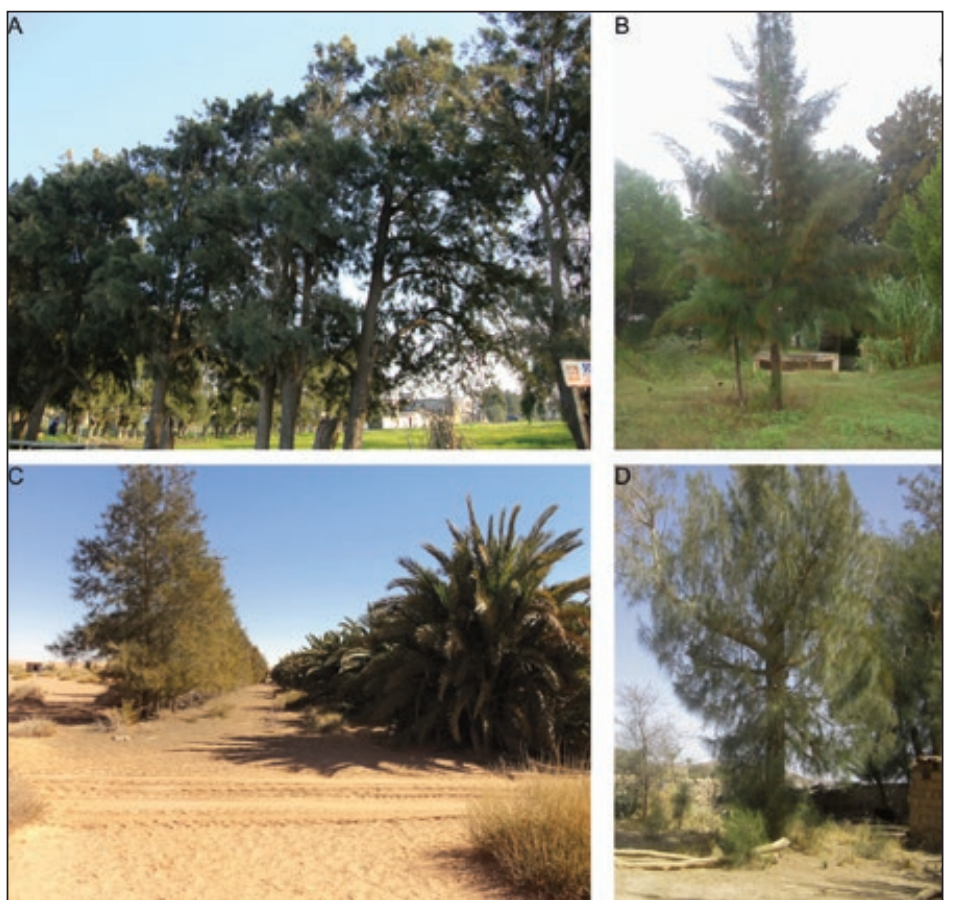

Photos 2.

Utilization of Casuarina trees in Algeria. A. Roadside delimitation by C. cunninghamiana in the coastal zone (El Kaddous, district of Algiers). B. C. cunninghamiana tree at the North region of Algeria (campus of Houari Boumedienne university, USTHB, district of Algiers).

C. Plantation of C. glauca as a windbreak in a palm grove in the South of the country (USTHB experimental field station, El-Goléa, district of Ghardaïa). D. C. glauca tree at the South of Algeria (District of Tamanrasset).

Photos K. Belaid. 


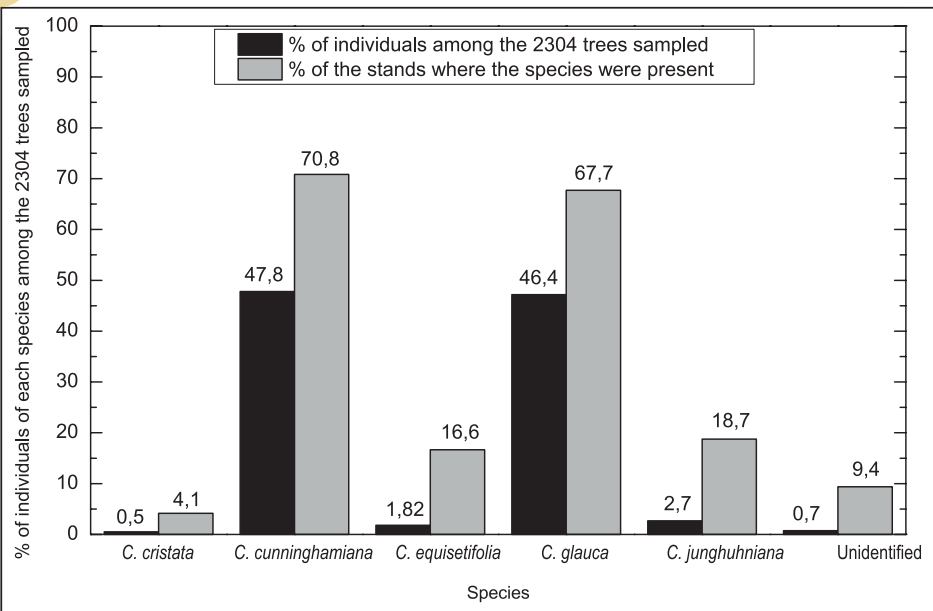

Figure 4.

Distribution of the 2,304 trees analyzed by species and proportion of stands where each species was present (all data are given as \% of the total individuals and \% of the 96 selected stands). Based on the $x^{2}$ tests, the proportion $\left(x^{2}{ }_{(11)}=205, p<0.001\right)$ of each species as well as their distribution $\left(x^{2}(5)=3850, p<0.001\right)$ in the stands were not homogeneous.

\section{Symbiotic status of the C. cunninghamiana and $C$. glauca seedlings grown in nurseries}

We examined whether seedlings of $C$. cunninghamiana and C. glauca produced in nurseries in Algeria are naturally mycorrhized and nodulated before their dissemination on the local market. For this, we assessed the prevalence of mycorrhizal and actinorhizal symbioses in 12-month-old seedlings produced in 12 nurseries chosen from different areas of Algeria.

\section{Prevalence of mycorrhization}

Careful examination of root systems of C. cunninghamiana and C. glauca plants did not reveal the presence of any ectomycorrhizal structures (Hartig net of hyphal mantle) indicating that these actinorhizal species were unable to form ectomycorrhizal symbiosis in the 12 nurseries from the North and the South of Algeria which regularly produce Casuarina seedlings for the local market (figure 1). In contrast, endomycorrhizal structures, mainly in the form of intra- and intercellular hyphae, were observed on most of the root systems of the two Casuarina species in all the nurseries included in the analysis. The presence of arbuscules and vesicles was also observed (photos $5 \mathrm{~A}$ and $5 \mathrm{~B}$ ). The percentage of mycorrhized plants ranged from $70.8 \%$ to $100 \%$ for C. cunninghamiana and from $83.3 \%$ to $100 \%$ for C. glauca (table II). However, seedlings growing in nurseries located in the South were less mycorrhized (table II). These results indicate that both species can interact with endomycorrhizal fungi.

The percentage of mycorrhized plants of C. cunninghamiana and C. glauca in the nurseries was high (from $86,5 \%$ to $93,8 \%$ ) while the mycorrhizal intensity (1\%) had an overall mean of about $53 \%$ for both species (table II). Furthermore, $1 \%$ was more intense in nurseries located in the North than those located in the South of the country (table II). However,

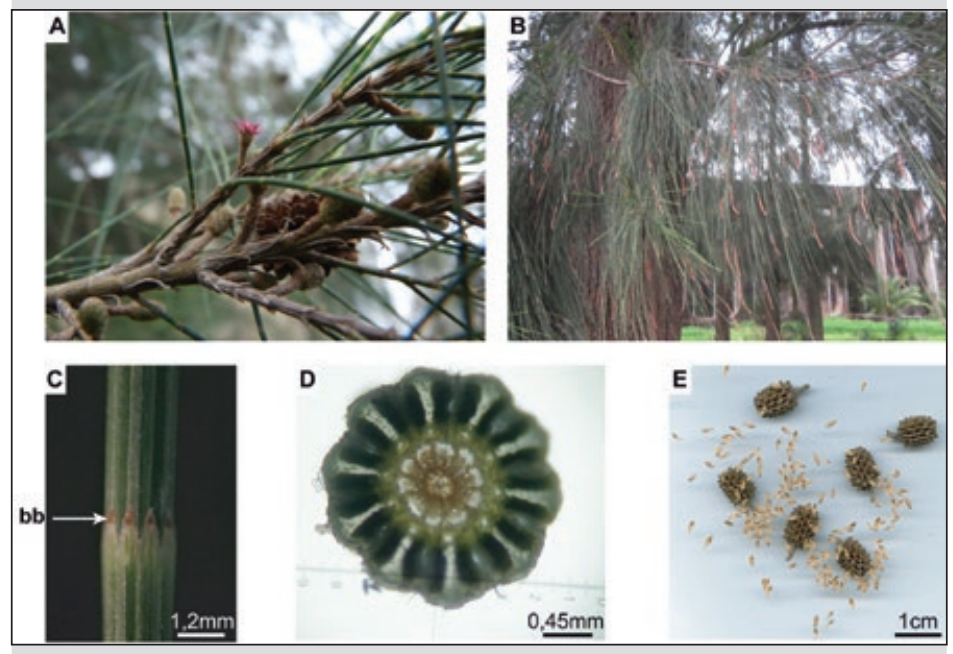

Photos 3.

Morphological characters of Casuarina cunninghamiana. A. Branches with female flowers; B. Branches with male flowers; C. Detail of a branch under a magnifying glass highlighting the leaves in whorls with small sharp teeth and a brown transverse band (bb); D. Cross section of a branch observed under a light microscope (10 teeth); E. Female cones and seeds. Photos K. Belaid.
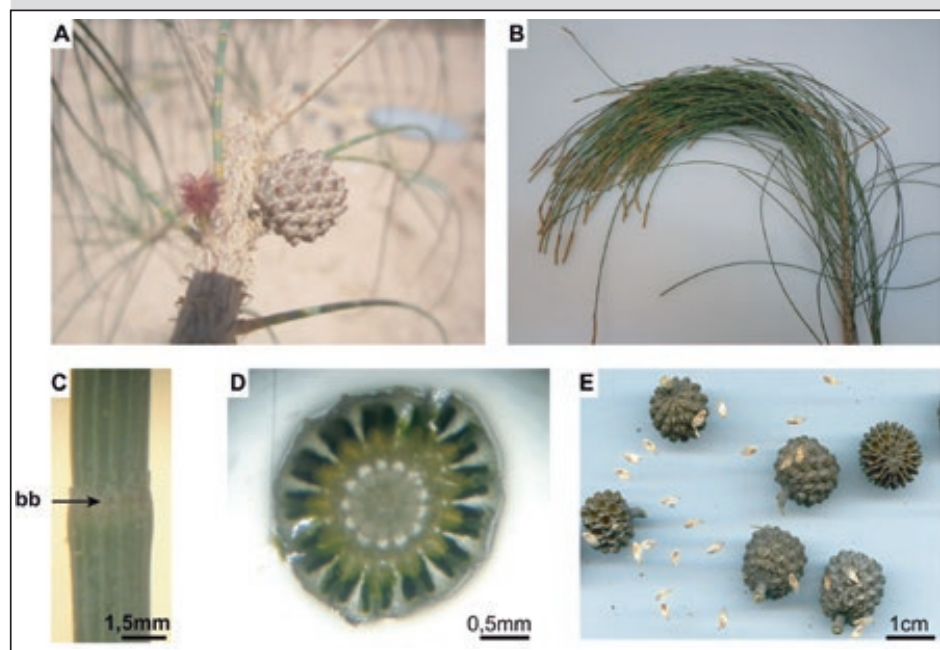

Photos 4.

Morphological characters of Casuarina glauca. A. Branches with female flowers; B. Branches with male flowers; C. Detail of a branch under a magnifying glass highlighting the leaves in whorls with small sharp teeth and a brown transverse band; D. Cross sections of a branch observed under a light microscope (13 teeth); E. Female cones and seeds. Photos K. Belaid.

in the nurseries in the North as well as in the South, I\% for both $C$. glauca and C. cunninghamiana seedlings were not significantly different (table II). 


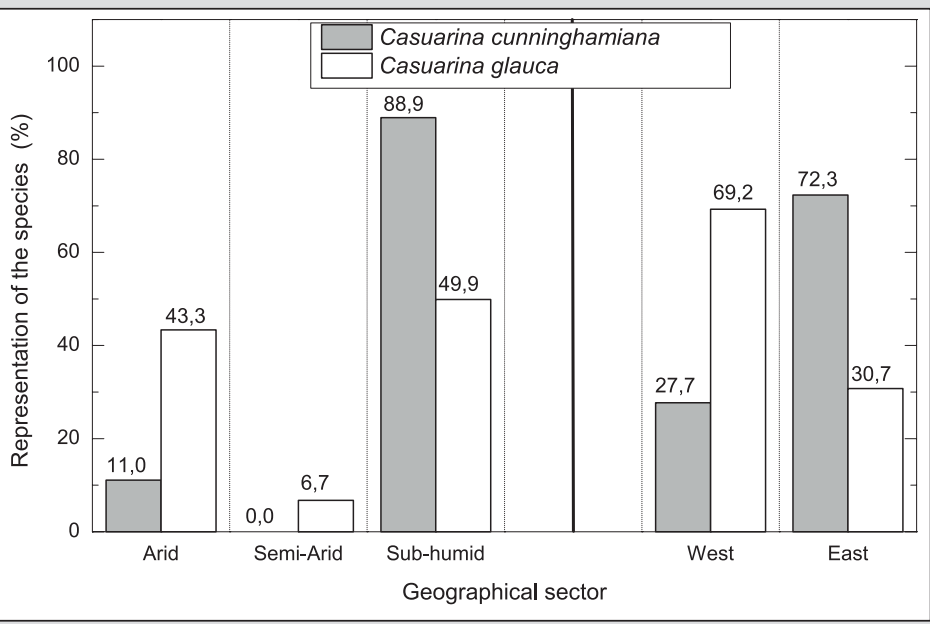

Figure 5.

Geographical distribution of the two dominant Casuarina species (data are given as $\%$ of the total individuals for each geographical zone)
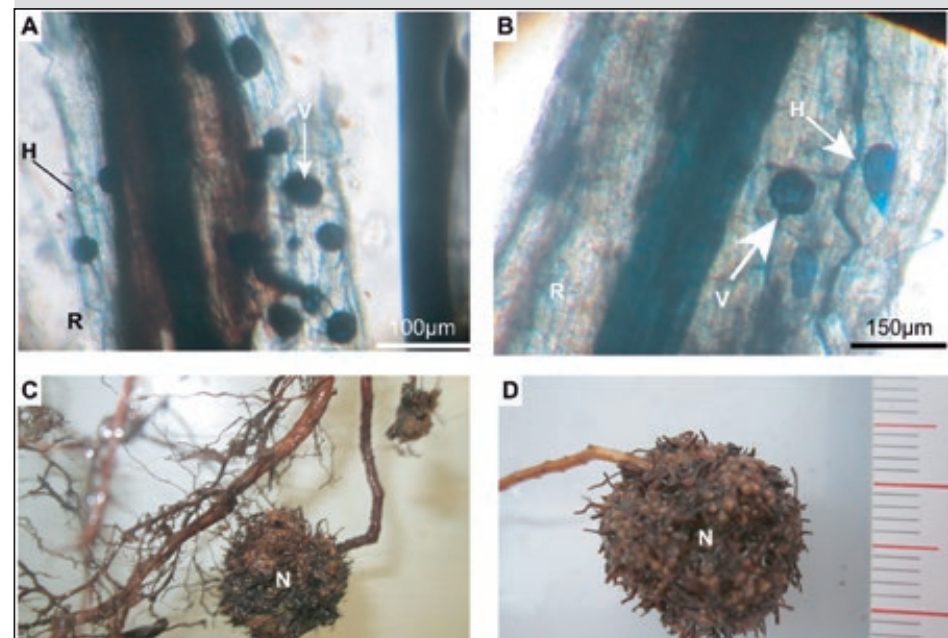

Photos 5.

Symbiotic status of Casuarina cunninghamiana and C. glauca. A. Light microscope photograph of arbuscular mycorrhizal (AM) colonization of the root of C. cunninghamiana showing intercellular hyphae in the root cortical tissue and vesicles in the cortical layer; B. Light microscope photograph of arbuscular mycorrhizal colonization of the root of $C$. glauca showing extraradical hyphae of AM attached with the root and vesicles in the cortical layer; C. Root nodules of C. cunninghamiana; D. Root nodules of C. glauca. H: hyphae; R: root; N: nodule; V: vesicle. Photos K. Belaid.

\section{Prevalence of nodulation}

Root systems of $C$. cunninghamiana and $C$. glauca seedlings were examined for the presence of nodules. Results indicate that a low proportion of seedlings produced in the 12 nurseries were nodulated (photos $5 C$ and 5D). The overall mean of nodulation was $28.8 \%$ for $C$. cunninghamiana and $26.4 \%$ for $C$. glauca with high differences between seedlings growing in northern and southern nurseries (table III). We have observed that seedlings growing in southern nurseries were much less nodulated ( $11.1 \%$ and $11.8 \%$ respectively) than those growing in northern nurseries $(46.5 \%$ and $50 \%$ respectively). In most cases, only a small fraction of seedlings was nodulated except for those growing in the nurseries of Algiers, Béjaia and El Kala located in a wetter zone. In addition, nodulation was absent in three nurseries located in the South (Districts of El Golea, Tamanrasset and Djanet). For both species, the number and dry weight of nodules per seedling were drastically lower in nurseries located in the South than those located in the North of the country. Overall, the two species showed a relatively reduced number of nodules and nodule dry weight per seedling (table III) and had a similar susceptibility to nodulation by Frankia in the 12 nurseries.

\section{Discussion}

Our survey conducted on 2,304 trees from 96 Casuarina stands situated all over Algeria indicates that Casuarina trees belong in most cases to the genus Casuarina. In Algeria, apart from 12 individuals likely to be Allocasuarina verticillata found in a unique site in the arboretum of Baïnem (district of Algiers), we did not find any records of Allocasuarina introduction listed in the literature: A. littoralis, A. paludosa and A. torulosa (GGA, 1850, 1865; Trottier, 1872; SGA, 1875; Toth, 1965). Our observations are consistent with those of Maire (1957) who stated that Allocasuarina species are very scarce in Algeria with A. verticillata (cited as Casuarina quadrivalvis) reported as the only species present.

Our investigations throughout the country showed that the genus Casuarina is represented by 5 species: C. cristata, C. cunninghamiana, C. equisetifolia, C. glauca and $C$. junghuhniana. However, individuals of $C$. cunninghamiana and C. glauca are by far the most frequent species in Algeria accounting for nearly $95 \%$ of the individuals sampled. These findings contradict previous reports considering $C$. equisetifolia as the most common or the only Casuarina species occurring in Algeria (Houmani, 1997). Our results are however partially in line with those of Maire (1957), who reported that only C. cunninghamiana and C. glauca occur in Algeria and stated that C. equisetifolia specimens previously reported from Algeria in the literature were in fact $C$. cunninghamiana, C. glauca or Allocasuarina verticillata (cited as Casuarina quadrivalvis). Such predominance of $C$. cunninghamiana and C. glauca is also observed in Morocco, Tunisia, and Egypt (El-Lakany, 1988; Ducousso et al., 2003; Tellal et al., 2008).

Furthermore, the two major species of Casuarina highlighted by this study showed a different distribution. Casuarina cunninghamiana is more common in the North and East which are the wettest and most temperate areas of the country while $C$. glauca is more represented in drier and warmer areas of the South and West (figure 5). Since there is no detailed history of the spread of Casuarina species after their introduction and establishment in Algiers, it is difficult to explain the origin of the current distribution of these 
two species throughout Algerian rangelands. However, this difference in their distribution can probably be linked to the ecological properties of the two species. Indeed, C. glauca species is characterized by its better tolerance to drought, salinity, frost, and high temperatures (El-Lakany and Luard, 1983) and would have been preferred for plantations in the driest and hottest parts of the country. Casuarina cunninghamiana is a more temperate species and less adapted to drought, salinity and high temperatures. This explains why C. cunninghamiana is preferably planted in coastal and wet areas of Algeria.

Amongst the list of species highlighted by this study, C. cristata has yet to be reported in Algeria or any other North-African country. Its presence should be considered with caution since the CDA resulted in a very scarce grouping of the 12 individuals we attributed to this taxon (figure 2). The misidentification of $C$. glauca as $C$. cristata could have occurred as their morphometric traits are remarkably similar (Wilson and Johnson, 1989). This confusion is common and has led many authors to mistakenly state that $C$. cristata occurs in areas in which it was never introduced such as in Florida, Caribbean Islands, and Taiwan (Woodal and Geary, 1985). A similar misidentification could have occurred by Beddiar et al. (2015) who describe C. obesa as present in the North-East of Algeria, a species for which no introduction history to the country exists.

In our study, the size of Casuarina samples (2,304 individuals) was large enough to limit misidentifications. However, one should not exclude the existence of atypical individuals or hybrids which are quite frequent in nature (El-Lakany and Yuness, 1995; Kamalakannan et al., 2006). In their native range of Australia, Casuarina species are typically allopatric - gene flow is limited by ecology (i.e., phenology and spatial distribution), rather than anatomical, biochemical or genetic incompatibilities (Woodall and Geary, 1985). Thus, crosses between species of the genus in which pollen is disseminated mainly by wind are possible as populations of Casuarina species with synchronous flowering periods overlap. Crossing between members of Casuarina genus has been observed in several areas of the world (Woodall and Geary, 1985; Wilson and Johnson, 1989).

Given that almost all Casuarina stands present in the country comprise two or more species and that the species present in Algeria all flower nearly simultaneously in early fall (September and October), the overlap of populations of different species may have favoured the appearance of many hybrids. The presence of hybrids in the stands we explored could explain why in almost all groups delimited by CDA, several individuals were ranged far from the centre of their corresponding cloud (figure 2). This could explain why we attributed some individuals to $C$. cristata, a species for which no introduction history in Algeria has been reported. This also could explain why a small number of individuals (17) could not be identified. Genetic markers could be used to confirm our identifications and to detect possible hybrids that can constitute an interesting plant genetic resource.

Regarding the symbiotic status of C. cunninghamiana and $C$. glauca, we observed that young individuals growing in each of the 12 nurseries were unable to deve- lop ectomycorrhizal symbiosis. By contrast, the two species showed susceptibility to endomycorrhizal fungi with a high proportion of individuals that were colonized (table II). Although Casuarina species can interact with both ectoand endomycorrhizal fungi, our observations are in line with previous reports indicating that they are more susceptible to endomycorrhizal fungi. Indeed, studies conducted by Mejstřík and Cudlín (1983), Nouasria and Beddiar (2010) and Beddiar et al., (2015) reported only endomycorrhizae for C. equisetifolia, C. cunninghamiana and C. obesa growing in Algeria. Furthermore, Duponnois et al. (2003) showed that under axenic conditions, isolates of the ectomycorrizal fungi Pisolithus and Scleroderma were unable to form a Hartig net on C. glauca while they could form ectomycorrhizae on Allocasuarina verticillata.

Although the endomycorrhization of Casuarina seedlings was globally high, the overall mycorrhizal intensity (I\%) was lower. This I\% does not exceed 53\% overall, which could be attributed to the reduced concentration of fungal inocula in culture substrates of the nurseries and/or to their composition. For instance, a high phosphate content of the substrate can strongly reduce or inhibit mycorrhization (Duponnois et al., 2003).

Concerning the nodulation by the actinobacteria Frankia, our investigations indicate that the presence of root nodules on C. cunninghamiana and C. glauca seedlings was scarce in the 12 nurseries studied. The overall proportion of nodulated seedlings was less than $30 \%$ with a reduced number of nodules and nodule dry weight per seedling (table III). These results are interesting as there is no information about the history of the inoculation of these trees in Algeria. It is known that generally Casuarina cannot be nodulated outside their natural range due to the absence of compatible Frankia strains (Reddell et al., 1986; Simonet et al., 1999). Nodulated plants observed in the different nurseries indicate that the soils contained viable Frankia strains able to nodulate the Casuarina plantlets. Since the specificity between Frankia and their Casuarina hosts results from a long co-evolution process between the two symbionts, it is unlikely that native actinobacteria have adapted to Casuarina, given their relatively recent introduction to the country. The most probable explanation for the presence of these compatible strains is their co-introduction by Casuarina plant material (seedlings, cones, seeds, and solid substrates). Once in Algeria, these strains persisted and spread through the country via local exchanges of plant material between central and regional nurseries. The extensive use of Casuarina in the country has likely contributed to the spread of actinobacteria (Houmani, 1997).

Nevertheless, overall, the seedlings were scarcely nodulated and showed reduced nodule numbers and nodule dry weights indicating that the symbiosis with Frankia was not very effective. This could result from different factors such as the presence of low concentrations of Frankia bacteria in the culture substrate in the nurseries (Reddell et al., 1986) or the chemical composition of the culture substrate. A phosphorus deficiency or a high content in nitrogen can also limit the nodulation of the seedlings (Dawson, 2008; Chaia et al., 2010). 
Furthermore, in the framework of this study, we could isolate a Frankia strain (named B2) from nodules collected from $C$. cunninghamiana seedlings growing in a nursery located at Souk El Tenine (District of Bejaia). The sequencing and the analysis of its genome indicate that the strain B2 is a new Algerian isolate (Belaid et al., 2020).

\section{Conclusion}

Since their introduction in Algeria at the beginning of the $19^{\text {th }}$ century, Casuarina trees have been rapidly propagated and widely planted for their rapid growth and hardiness and have become an important part of the ligneous flora of the country.

Our analysis revealed that the Casuarina trees currently occurring in the country belong to only 5 species with an overwhelming representation (95\%) of the 2 species C. cunninghamiana and C. glauca which displayed distinct distribution. Casuarina cunninghamiana is more common in the North and the East while C. glauca is more commonly found in the South and the West. In addition, we found that $C$. cunninghamiana and $C$. glauca seedlings produced in nurseries showed low rates of spontaneous mycorrhizal intensity and nodulation. This can have an impact in the successful establishment of the trees in the planting areas where soils are poor or degraded.

Finally, our survey of Casuarina in Algeria brings new data and confirms with greater accuracy not only the identity but also the relative abundance and the distribution of a globally important tree genus. Further work is needed to evaluate the presence of putative hybrids (e.g., using genetic markers for the analysis of the morphometric traits), which can have implications for their use as commercially important trees. Similar works are needed in other countries where Casuarina have been widely planted, especially in the neighbouring countries in North Africa. This will allow to have an inventory of their distribution and to assess their ecological impact in this region.

\section{References}

APG-The Angiosperm Phylogeny Group, Chase M. W., Christenhusz M. J. M., Fay M. F., Byng J. W., Judd W. S., et al., 2016. An update of the Angiosperm Phylogeny Group classification for the orders and families of flowering plants: APG IV. Botanical Journal of the Linnean Society, 181: 1-20. https://doi.org/10.1111/boj.12385

Beddiar A., Adouane M., Boudiaf I., Fraga F., 2015. Mycorrhizal status of main spontaneous or introduced forest trees in El Tarf province (Algerian North-east). The Online Journal of Science and Technology, 5 (2): 40-45. https://dergipark.org.tr/tr/download/article-file/210262 Benge M. D., 1982. Casuarinas, "the best firewood in the world": Resources for charcoal, construction poles, windbreaks and shelterbelts and soil erosion and sand dune stabilization. Washington, DC, USA, Agency for International Development, $110 \mathrm{p}$.

Belaid K., Swanson E., Carré-Mlouka A., Hocher V., Svistoonoff S., Simpson S., et al., 2020. Draft genome sequence of the symbiotic Frankia sp. strain B2 isolated from root nodules of Casuarina cunninghamiana found in Algeria. Journal of Genomics, 8: 11-15. https:// doi.org/10.7150/igen.38461
Bensaid S., 1995. Bilan critique du barrage vert en Algérie. Sécheresse, 3 (6): 247-255.

Chaia E. E., Wall L. G., Huss-Danell K., 2010. Life in soil by the actinorhizal root nodule endophyte Frankia: A review. Symbiosis, 51 (3): 201-206. https://doi.org/10.1007/s13199-010-0086-y

Christenhusz M. J. M., Byng J. W., 2016. The number of known plants species in the world and its annual increase. Phytotaxa, 261 (3): 201217. https://doi.org/10.11646/phytotaxa.261.3.1

Cruz-Castillo J. G., Ganeshanandam S., MacKay B. R., Lawes G. S., Lawoko C. R. O., Woolley D. J., 1994. Applications of canonical discriminant analysis in horticultural research. HortScience, 29 (10): 11151119. https://doi.org/10.21273/hortsci.29.10.1115

Dawson J. O., 2008. Ecology of actinorhizal plants. In: Pawloski K., Newton W. E. (eds). Nitrogen fixation: origins, applications, and research progress. Vol. 6: Nitrogen-fixing actinorhizal symbioses. Dordrecht, Netherlands, Springer, 199-234.

Ducousso M., Arahou M., Nourissier-Mountou S., Echbab H., Tellal M., Prin Y., et al., 2003. Les microorganismes symbiotiques associés aux racines de Casuarina Cunninghamiana et de Casuarina glauca au Maroc. Annales de la Recherche Forestière au Maroc, 36 : 9-25.

Duponnois R., Diédhiou S., Chotte J. L., Sy M. O., 2003. Relative importance of the endomycorrhizal and (or) ectomycorrhizal associations in Allocasuarina and Casuarina genera. Canadian Journal of Microbiology, 49 (4): 281-287. https://doi.org/10.1139/w03-038

El-Lakany M. H., 1988. Experience with some Australian species as multi-purpose trees in Egypt and North African countries. In: Proceedings of International forestry conference for the Australian bicentenary, Albury Wodonga, Australia, $25^{\text {th }}$ April - $1^{\text {st }}$ May 1988 . Vol. V. Albury, Wodonga, Australia, Australian Forestry Development Institute, 16-33.

El-Lakany M. H., Luard E. J., 1983. Comparative salt tolerance of selected Casuarina species. Australian Forestry Research, 13: 11-20.

El-Lakany M. H., Yuness M. I., 1985. Genetic variation in some morphological and growth characteristics in Casuarina cunninghamiana and C. glauca. In: Eguiluz-Piedra T. (ed.). The State of the Art in Forest Genetics and Breeding. North American Forestry Commission, Universidad Autónoma Chapingo, Mexico, 143 p.

GGA (Gouvernement Général de l'Algérie), 1850. Catalogue des végétaux cultivés à la pépinière centrale du gouvernement à Alger. Imprimerie du Gouvernement, Alger, 107 p.

GGA (Gouvernement Général de l'Algérie), 1865. Catalogue des végétaux et graines disponibles et mis en vente au jardin d'acclimatation au Hamma (près d'Alger) pendant l'automne 1865 et le printemps 1866. Bastide, Libraire Éditeur, Alger. $152 \mathrm{p}$.

Gherbi H., Diagne N., Duponnois R., Bogusz D., Franche C., Bekki A., 2011. Casuarinaceae for soil rehabilitation in Algeria. In: Zhong C., Pinyopusarerk K., Kalinganire A., Franche C. (eds). Improving smallholder livelihoods through improved Casuarina productivity. Proceedings of the 4th International Casuarina Workshop, Haikou, China, 21-25 March 2010. China Forestry Publishing House, 249 p.

Hammer $\emptyset$., Harper D. A. T., Ryan P. D., 2001. PAST: Paleontological Statistics Software Package for Education and Data Analysis. Palaeontologia Electronica, 4 (1): 1-9. https://palaeo-electronica. org/2001_1/past/issue1_01.htm

Houmani Z., 1997. Multiplication and utilization of ornamental trees in central Algeria. In: Heywood V. H., Skoula M. (eds). Identification of wild food and non-food plants of the Mediterranean region. Chania, Greece, CIHEAM, 33-42.

Johnson L. A. S., Wilson K. L., 1993. Casuarinacea. In: Kubitzki K., Rohwer J. G., Bittrich V. (eds). The families and genera of vascular plants. Volume II. Flowering plants - Dicotyledons. Magnoliid, Hamamelid and Caryophyllid families. Berlin, Germany, Springer Verlag, 237-242. 
Kamalakannan R., Barthwal S., Chezhian P., Balasaravanan T., Yasodha R., Gurumurthi K., Ghosh M., 2006. Morphological and molecular diversity among Casuarina and Allocasuarina species. Biotechnology, 5 (3): 301-307. https://scialert.net/abstract/?doi=biotech.2006.301.307

Maire R., 1957. Flore de l'Afrique du Nord : (Maroc, Algérie, Tunisie, Tripolitaine, Cyrénaïque et Sahara). Volume IV, Monocotyledonae : glumiflorae : Cyperaceae, Principes, Spathiflorae, Commelinales. In : L'Encyclopédie biologique. Vol. IV. Paris, France, Éditions Paul Lechevalier, $333 \mathrm{p}$.

Maity P. J., Pawlowski K., 2021. Anthropogenic influences on the distribution of the Casuarina-Frankia symbiosis. Symbiosis, 84: 353-367. https://doi.org/10.1007/s13199-021-00765-5

Makhlouf L., Nedjahi A., Abdellaoui M., Benarar S., 2012. Protection des périmètres agricoles dans les régions arides et semi-arides. Alger, Institut National de la Recherche Forestière, $44 \mathrm{p}$.

Mejstřik V. K., Cudlín P., 1983. Mycorrhiza in some desert plant species in Algeria. Plant and Soil, 71: 363-366.

Nouasria D., Beddiar A., 2010. Le statut symbiotique de Casuarina equisetifolia (filao) dans le Nord-Est Algérien. Biodiversité, physiologie et écologie. Tunisian Journal of Medicinal Plants and Natural Products, 3: 77-83.

NRC (National Research Council), 1984. Casuarinas: nitrogen-fixing trees for adverse sites. Washington, DC, USA, National Academy Press, $118 p$

Phillips J. M., Hayman D. S., 1970. Improved procedures for clearing roots and staining parasitic and vesicular-arbuscular mycorrhizal fungi for rapid assessment of infection. Transactions of the British Mycological Society, 55 (1): 158-161. https://doi.org/10.1016/S00071536(70)80110-3

Potgieter L. J., Richardson D. M., Wilson J. R. U., 2014. Casuarina: biogeography and ecology of an important tree genus in a changing world. Biological Invasions, 16 (3): 609-633. https://doi.org/10.1007/ s10530-013-0613-x

Rakotomalala R., 2005. TANAGRA: un logiciel gratuit pour l'enseignement et la recherche. In: EGC 2005, vol. RNTI-E-3, 697-702.

R Development Core Team, 2016. R: A Language and Environment for Statistical Computing. Vienna, Austria, R Foundation for Statistical Computing. https://www.R-project.org/

Reddell P., Bowen G. D., Robson M., 1986. Nodulation of Casuarinaceae in relation to host species and soil properties. Australian Journal of Botany, 34: 435-444.

SGA (Société Générale Algérienne), 1875. Jardin du Hamma (près d'Alger) - Végétaux et graines - Catalogue général $n^{\circ} 3$. Imprimerie horticole de E. Donnaud, Paris, $160 \mathrm{p}$.

Simonet P., Navarro E., Rouvier C., Reddell P., Zimpfer J., Dommergues Y., et al., 1999. Co-evolution between Frankia populations and host plants in the family Casuarinaceae and consequent patterns of global dispersal. Environmental Microbiology, 1 (6): 525-533.

Tarai N., Doumandji S., 2009. Feeding preferences of gregarious nymphs and adults of the Desert locust, Schistocerca gregaria Forskal (Orthoptera, Cyrtacanthacridinae) in different habitats at Biskra oasis, Algeria. Advances in Environmental Biology, 3 (3): 308-313.

Tellal M., Qarro M., Arahou M., Abourouh M., Tellal R., Rhazi L., et al., 2008. Effet de l'actinomycète Frankia sur la croissance et la fixation de l'azote de Casuarina glauca et Casuarina cunninghamiana. Sécheresse, 19 (3) : 211-216.

Toth J., 1965. Aspect forestier d'une plantation saharienne. Revue Forestière Française, 10 : 674-695.

Trottier M., 1872. Les arbres de l'Australie. Typographie de l'association ouvrière Aillaud et Compagnie, Alger, $20 \mathrm{p}$.

Trouvelot A., Kough J. L., Gianinazzi-Pearson V., 1986. Mesure du taux de mycorhization VA d'un système radiculaire. Recherche de méthode d'estimation ayant une signification fonctionnelle. In: Gianinazzi-Pearson V., Gianinazzi S. (eds). Physiology and genetics aspects of Mycorrizae. Paris, France, INRA, 217-221.
Wall L. G., 2000. The actinorhizal symbiosis. Journal of Plant Growth Regulation, 19: 167-182.

Wheeler G. S., Taylor G. S., Gaskin J. F., Purcell M. F., 2011. Ecology and management of Sheoak (Casuarina spp.), an invader of coastal Florida, USA. Journal of Coastal Research, 27: 485-492. https://doi. org/10.2112/JCOASTRES-D-09-00110.1

Wilson K. L., Johnson L. A. S., 1989. Casuarinaceae. In: George A. S. (ed.). Flora of Australia. 3. Hamamelidales to Casuarinales. Canberra, Australia, Australian Government Publishing Service, 100-174.

Woodall S. L., Geary T. F., 1985. Identity of Florida Casuarinas. USDA, Forest Service, Southeastern Forest Experiment Station, Research Note, 10-12.

Zhong C., Zhang Y., Chen Y., Jiang Q., Chen Z., Liang J., et al., 2010. Casuarina research and applications in China. Symbiosis, 50: 107-114. https://doi.org/10.1007/s13199-009-0039-5

\begin{tabular}{l|l|}
$\begin{array}{l}\text { Belaïd } \text { et al. - Author's contributions } \\
\text { Contributor role } \\
\text { Conceptualization }\end{array}$ & $\begin{array}{l}\text { Contributor names } \\
\text { S. Amrani, H. Gherbi }\end{array}$ \\
\hline Data Curation & S. Amrani, K. Belaid, H. Gherbi \\
\hline Formal Analysis & S. Amrani, K. Belaid, H. Gherbi \\
\hline Funding Acquisition & S. Amrani, H. Gherbi \\
\hline Investigation & K. Belaid, M. Zizi, S. Amrani, H. Gherbi \\
\hline Methodology & S. Amrani, K. Belaid, H. Gherbi \\
\hline Project Administration & S. Amrani, K. Belaid, M. Zizi, H. Gherbi \\
\hline Resources & S. Amrani, K. Belaid, M. Zizi, H. Gherbi \\
\hline Supervision & S. Amrani, H. Gherbi \\
\hline \begin{tabular}{l} 
Validation \\
\hline Visualization
\end{tabular} & S. Amrani, K. Belaid, M. Zizi, H. Gherbi \\
\hline Writing - Original Draft \\
Preparation
\end{tabular}

\section{Funding}

This study was funded by the Algerian Ministry of Higher Education and Scientific Research (MESRS). Kathia Belaid received a fellowship from MESRS to carry out a part of her PhD work in the LSTM laboratory (IRD/CIRAD/INRAe/ Montpellier University/Supagro, Campus de Baillarguet, Montpellier, France).

\section{Data access}

Data collected as part of the work summarised in the article submitted to Tropical Timber and Forests (Excel files) may be used by contacting the corresponding author and citing the article. They are publicly available: https://drive.google.com/drive/ folders/18DGeFPOYfQb68adI4kjudBSOtMjetKSo?usp=sharing

Bois et Forêts des Tropiques - Revue scientifique du Cirad (c) Bois et Forêts des Tropiques @ C Cirad

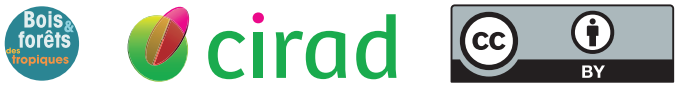

Cirad - Campus international de Baillarguet,

34398 Montpellier Cedex 5, France

Contact : bft@cirad.fr - ISSN : L-0006-579X 